\title{
The Photodecarboxylation of Substituted Naphthylmethyl Arylacetate Esters: Synthesis of Naphthylarylethanes
}

\author{
James W. Hilborn, ${ }^{\dagger *}$ Reinaldo Moya-Barrios ${ }^{\S}$ and Alison Thompson ${ }^{\S *}$ \\ ${ }^{\dagger} 2$ Wilewood Dr., R.R.\#2, Falmouth, Nova Scotia, B0P 1L0, Canada \\ ${ }^{\S}$ Department of Chemistry, Dalhousie University, P.O. Box 15000, Halifax, Nova Scotia, B3H \\ 4R2, Canada \\ Alison.Thompson@dal.ca
}

\section{Supporting Information}

\section{Table of contents}

NMR spectra

1-Naphthylmethyl 1-naphthylacetate (1a) ……………………………………………………..S2

1-Naphthylmethyl 2-naphthylacetate (1b) ......................................................................................S3

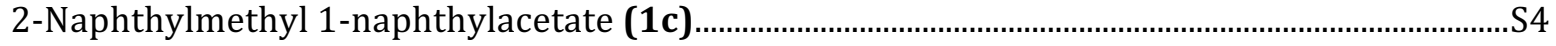

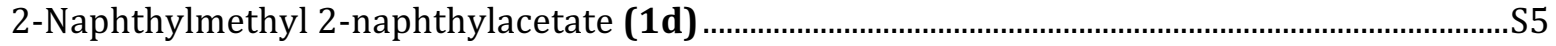

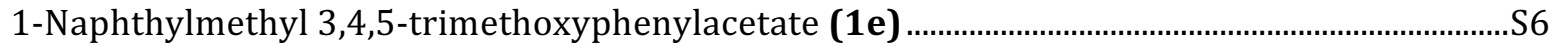

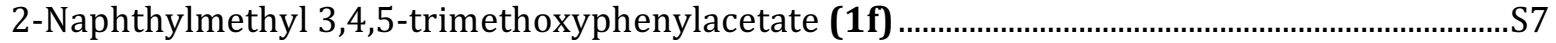

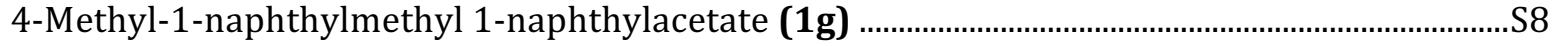

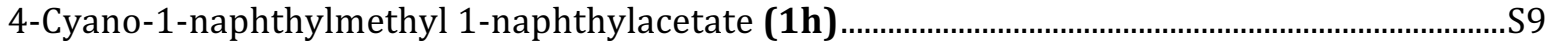

4-Cyano-1-naphthylmethyl 3,4,5-trimethoxyphenylacetate (1i) ...............................................SP10

1-(1-Naphthyl)-2-(1-naphthyl)ethane (2a) …………………...................................................S11

1-(1-Naphthyl)-2-(2-naphthyl)ethane (2b/c) ............................................................................. 512

1-(2-Naphthyl)-2-(2-naphthyl)ethane (2d) ………………………………………………….....S13

1-(1-Naphthyl)-2-(3,4,5-trimethoxyphenyl)ethane (2e) ........................................................... S14

1-(2-Naphthyl)-2-(3,4,5-trimethoxyphenyl)ethane (2f) …………………………………………...S15

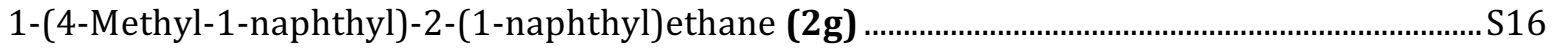

1-(4-Cyano-1-naphthyl)-2-(1-naphthyl)ethane (2h) ………….............................................S17

1-(4-Cyano-1-naphthyl)-2-(3,4,5-trimethoxyphenyl)ethane (2i).............................................S18

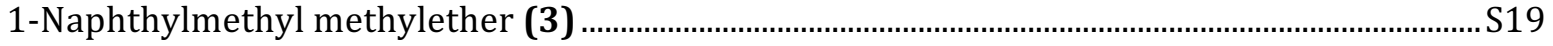

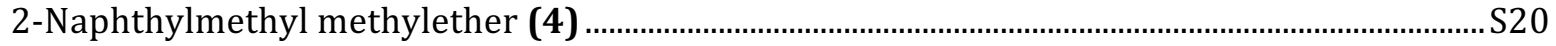

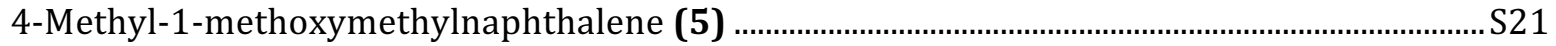

4-Methoxymethyl-1-naphthonitrile (6) ..................................................................................... S22 


\section{NMR spectra}

1-Naphthylmethyl 1-naphthylacetate (1a)

${ }^{1} \mathrm{H}-\mathrm{NMR}\left(500 \mathrm{MHz}, \mathrm{CDCl}_{3}\right)$

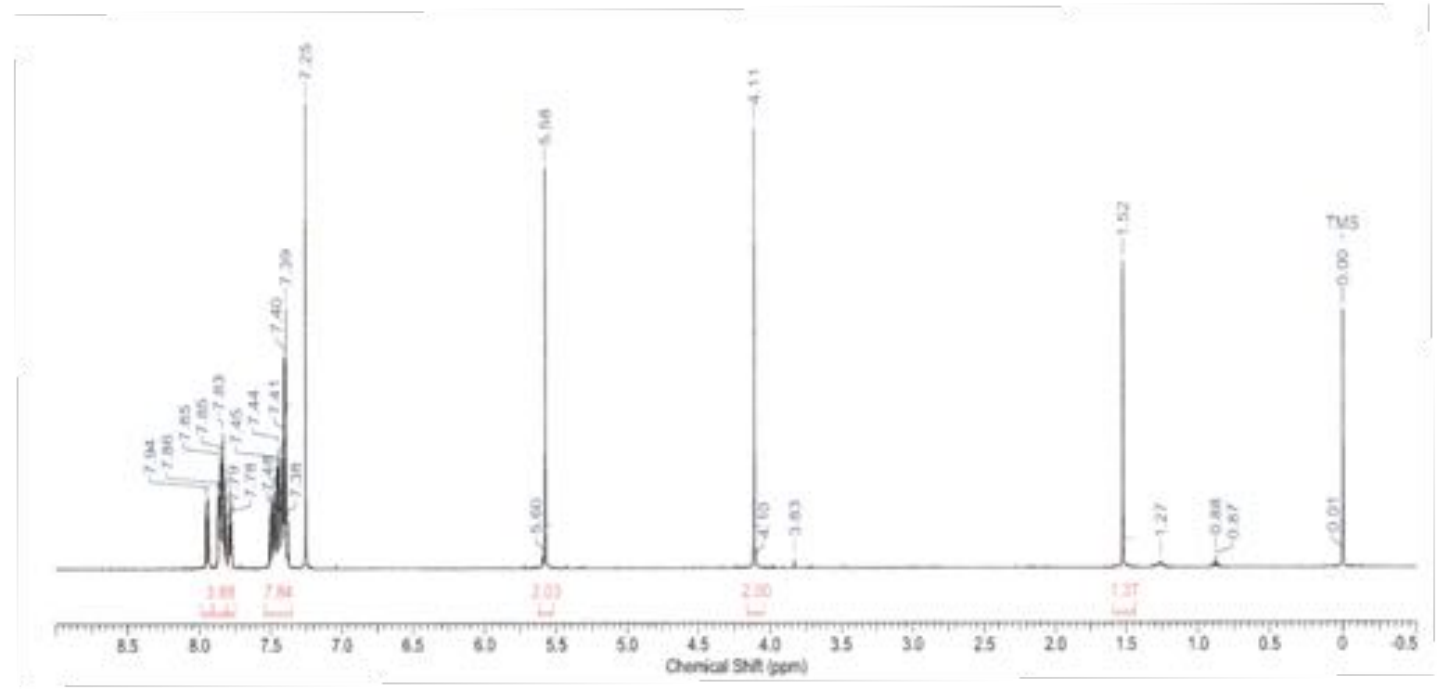

${ }^{13} \mathrm{C}$ UDEFT NMR (125 MHz, $\mathrm{CDCl}_{3}$ )

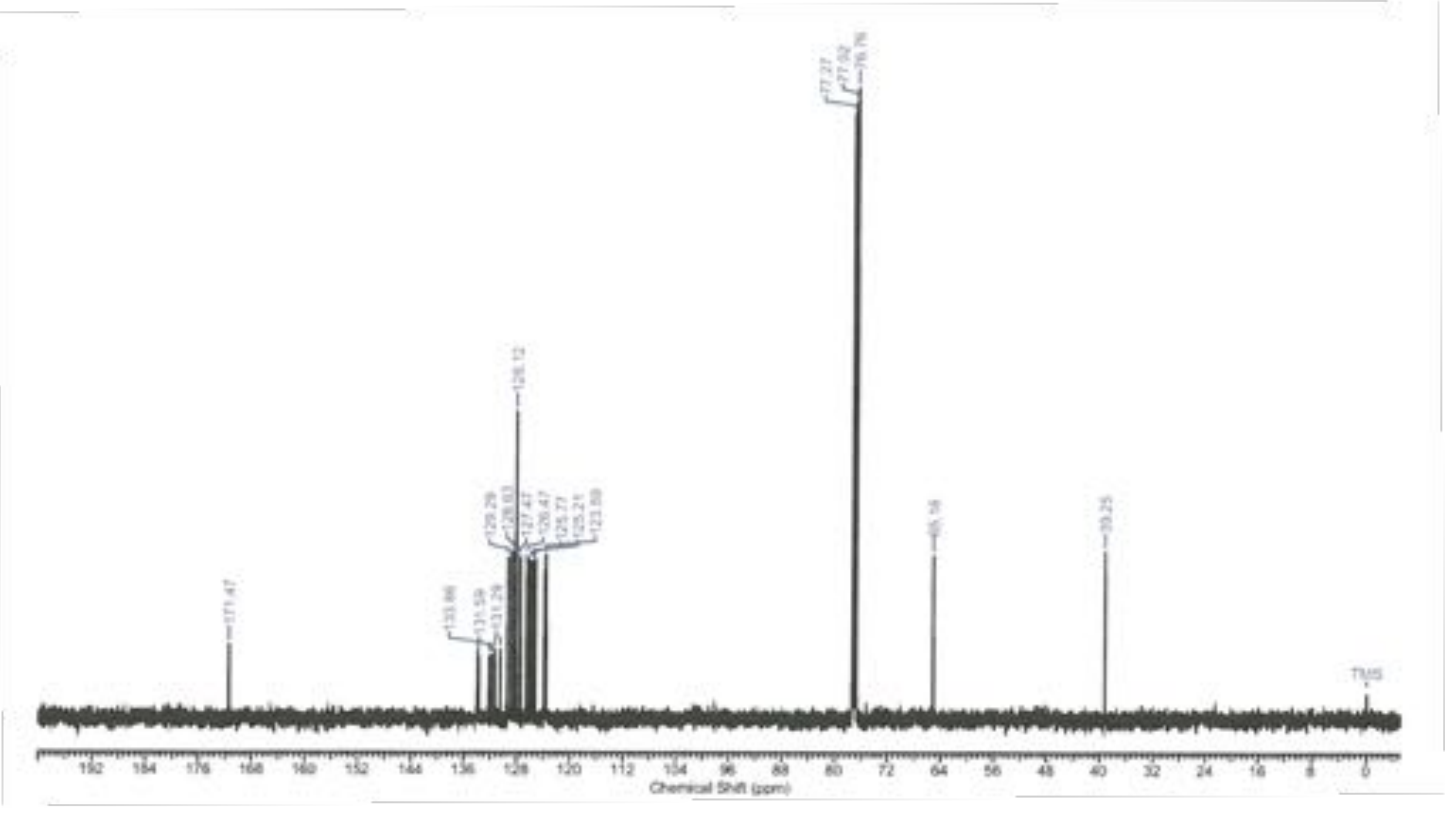


1-Naphthylmethyl 2-naphthylacetate (1b)

${ }^{1} \mathrm{H}-\mathrm{NMR}\left(500 \mathrm{MHz}, \mathrm{CDCl}_{3}\right)$

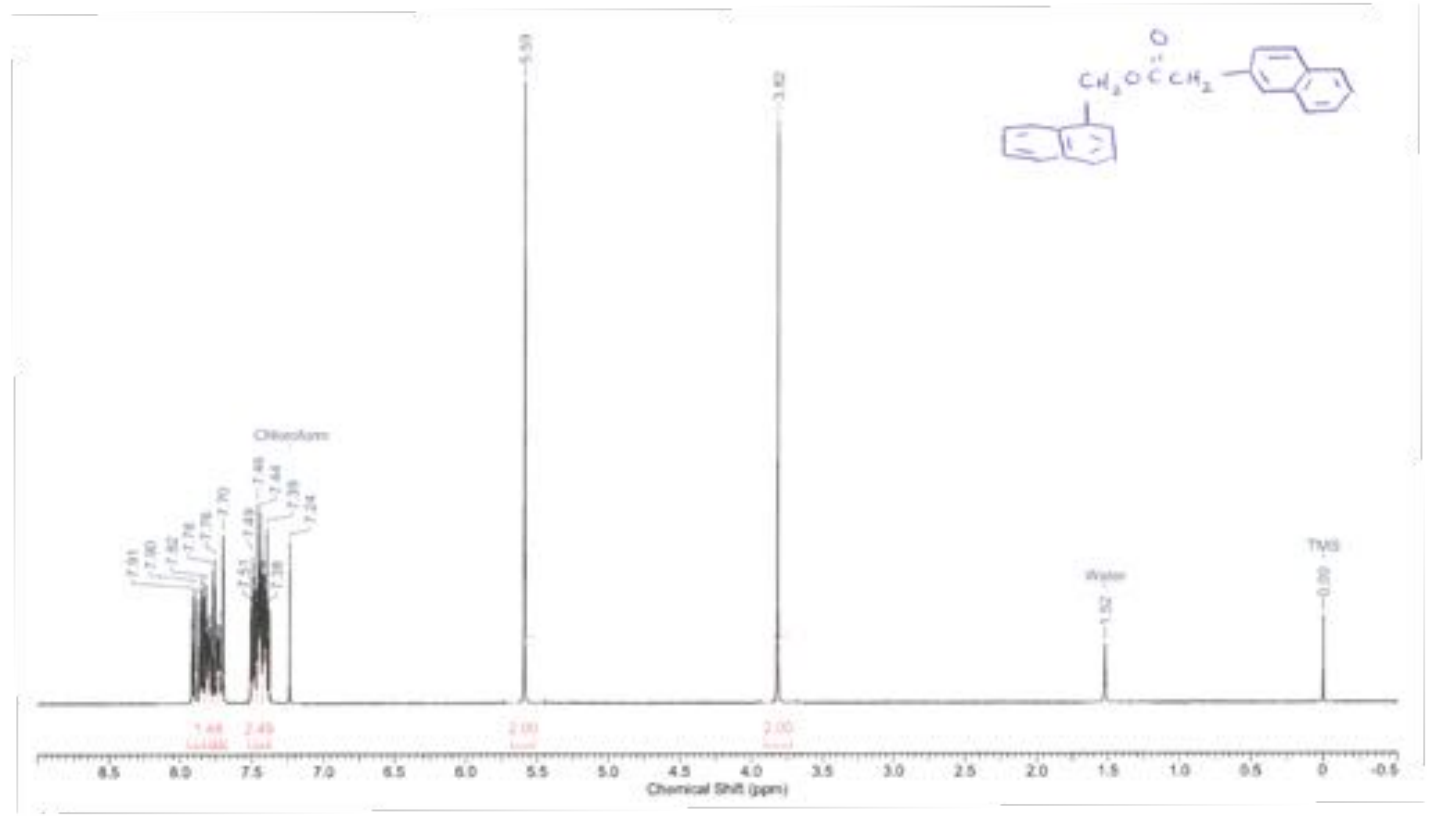

${ }^{13} \mathrm{C}$ UDEFT NMR (125 MHz, $\left.\mathrm{CDCl}_{3}\right)$

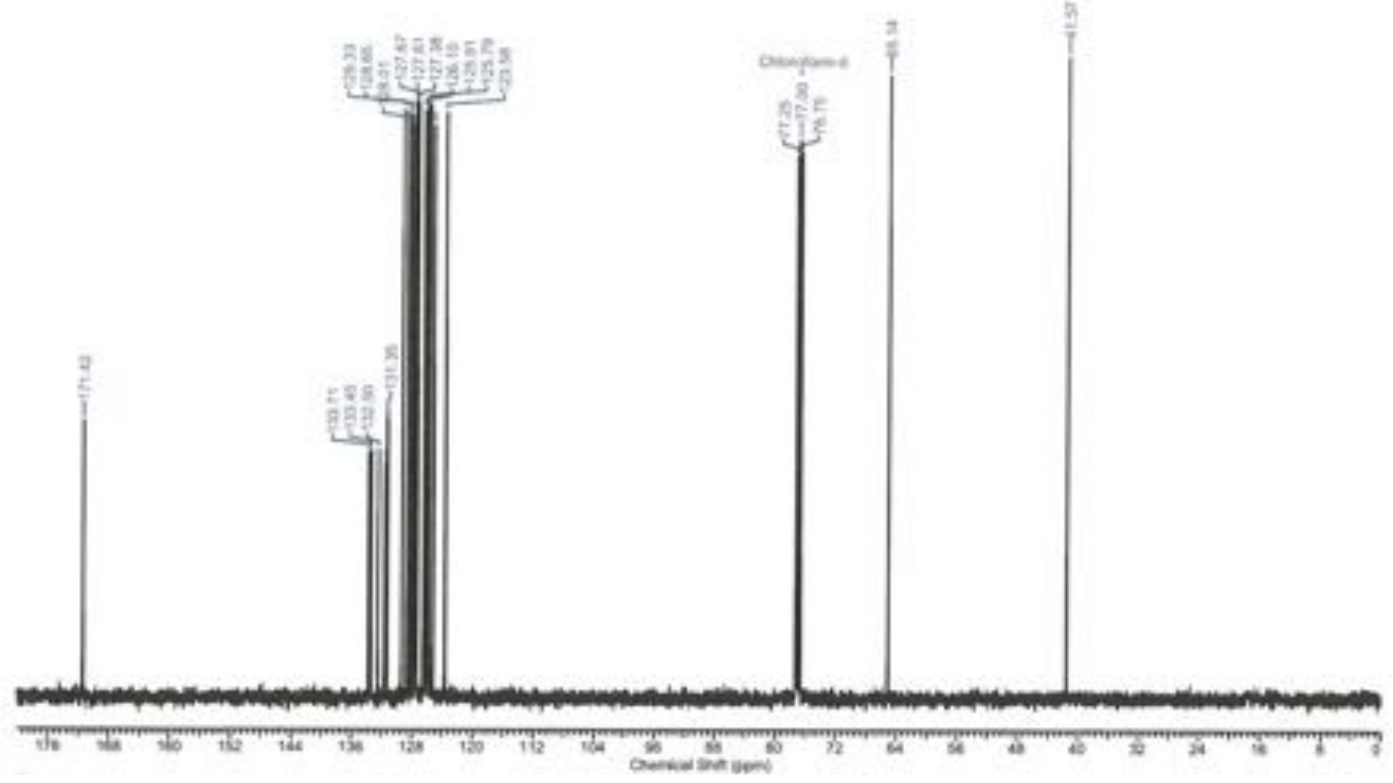


2-Naphthylmethyl 1-naphthylacetate (1c)

${ }^{1} \mathrm{H}-\mathrm{NMR}\left(500 \mathrm{MHz}, \mathrm{CDCl}_{3}\right)$

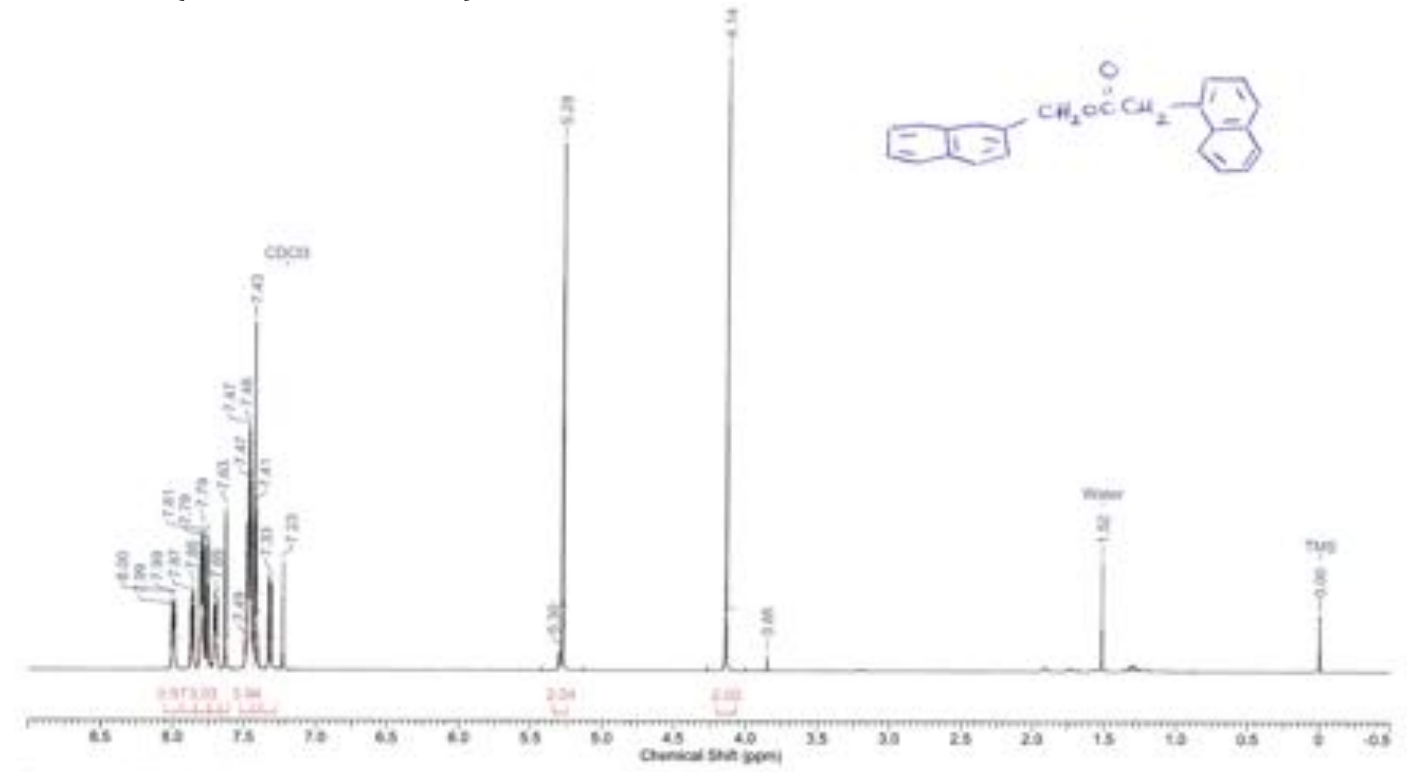

${ }^{13} \mathrm{C}$ UDEFT NMR (125 MHz, $\left.\mathrm{CDCl}_{3}\right)$

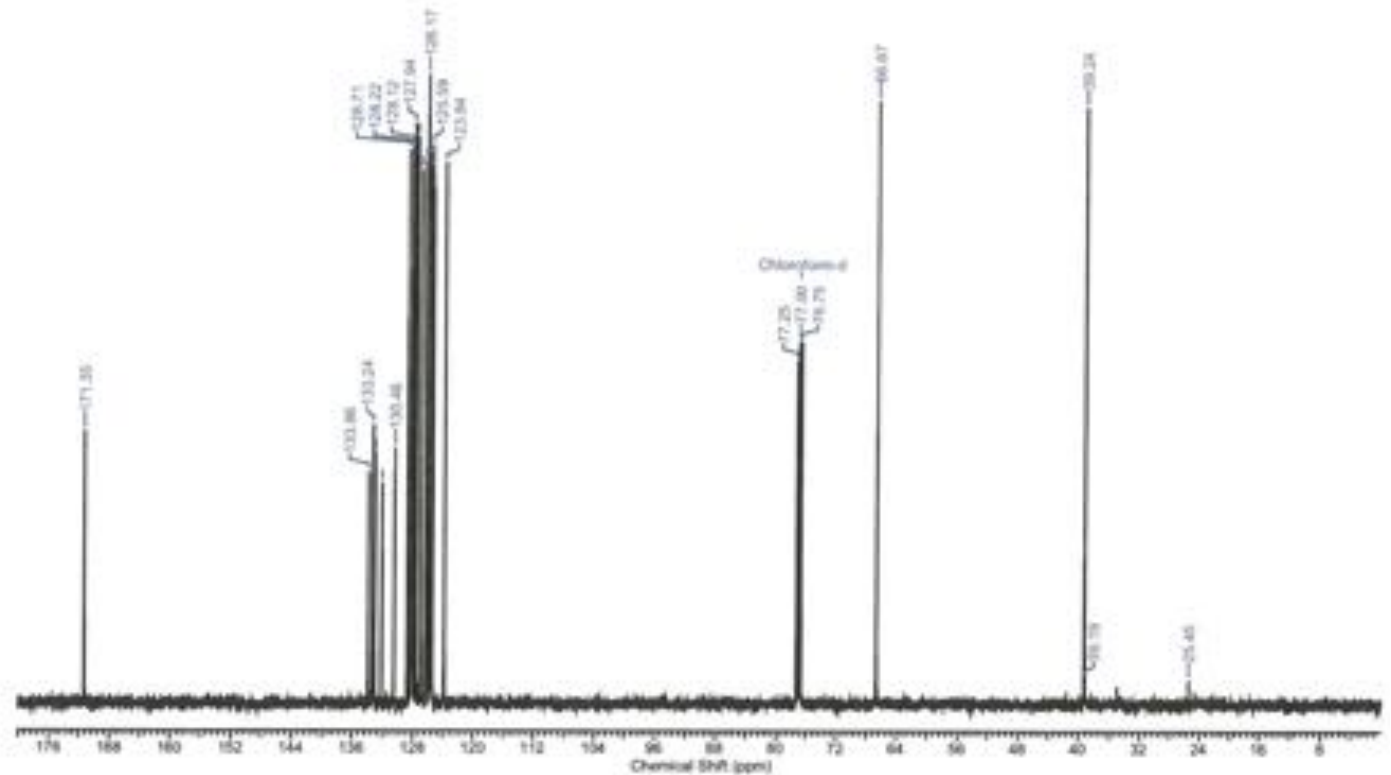


2-Naphthylmethyl 2-naphthylacetate (1d)

${ }^{1} \mathrm{H}-\mathrm{NMR}\left(500 \mathrm{MHz}, \mathrm{CDCl}_{3}\right)$

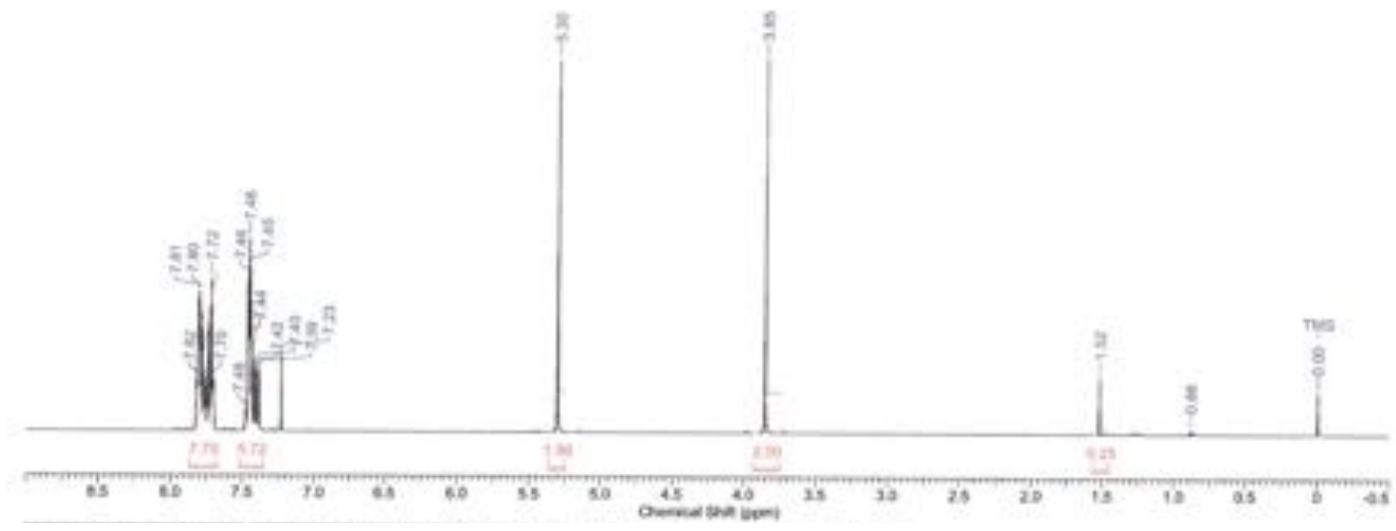

${ }^{13} \mathrm{C}$ UDEFT NMR (125 MHz, $\left.\mathrm{CDCl}_{3}\right)$

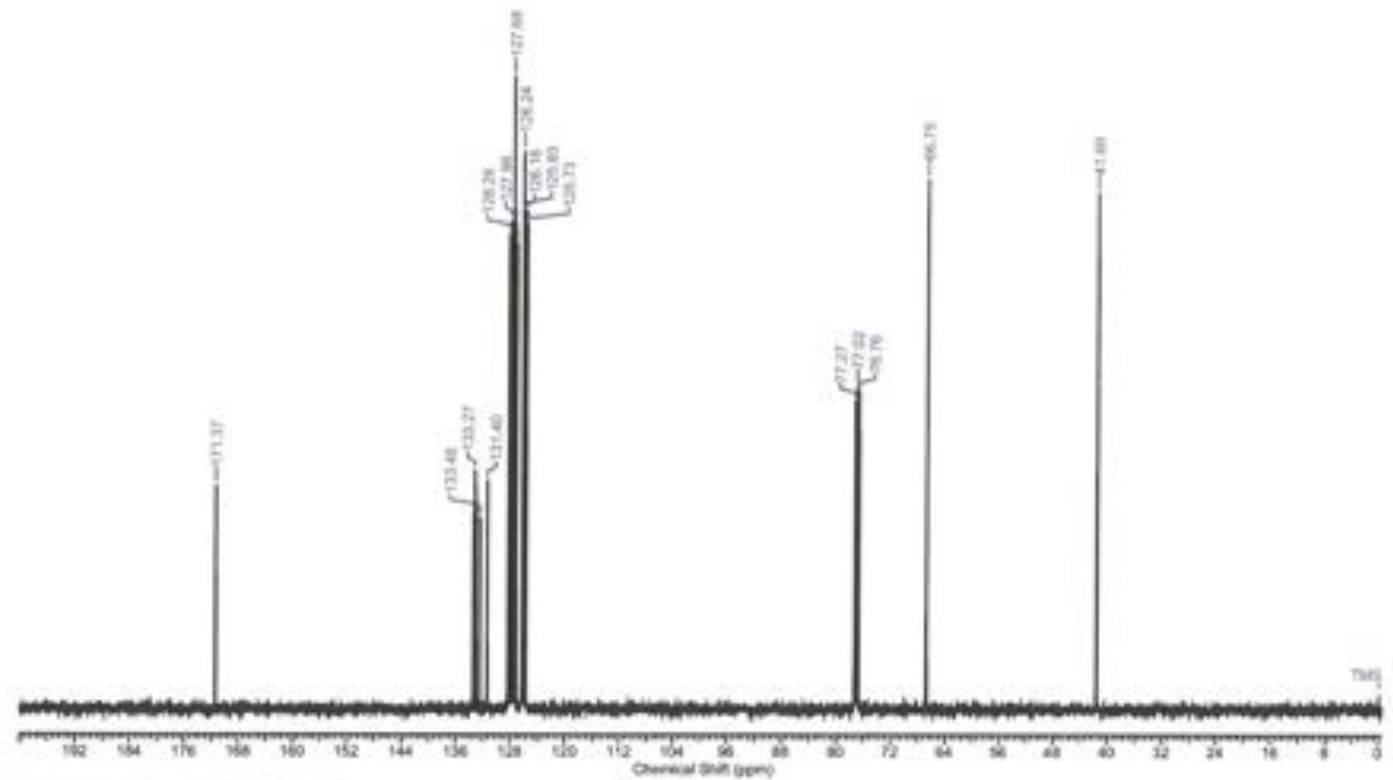


1-Naphthylmethyl 3,4,5-trimethoxyphenylacetate (1e)

${ }^{1} \mathrm{H}-\mathrm{NMR}\left(500 \mathrm{MHz}, \mathrm{CDCl}_{3}\right)$

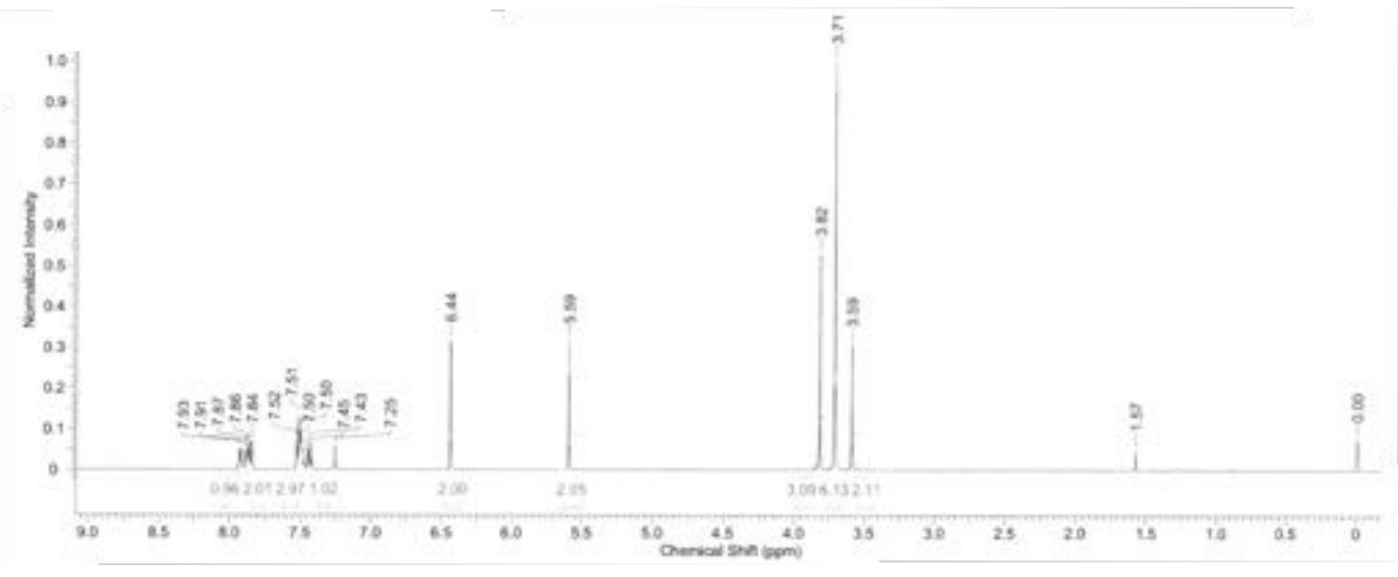

${ }^{13} \mathrm{C}$ UDEFT NMR (125 MHz, $\left.\mathrm{CDCl}_{3}\right)$

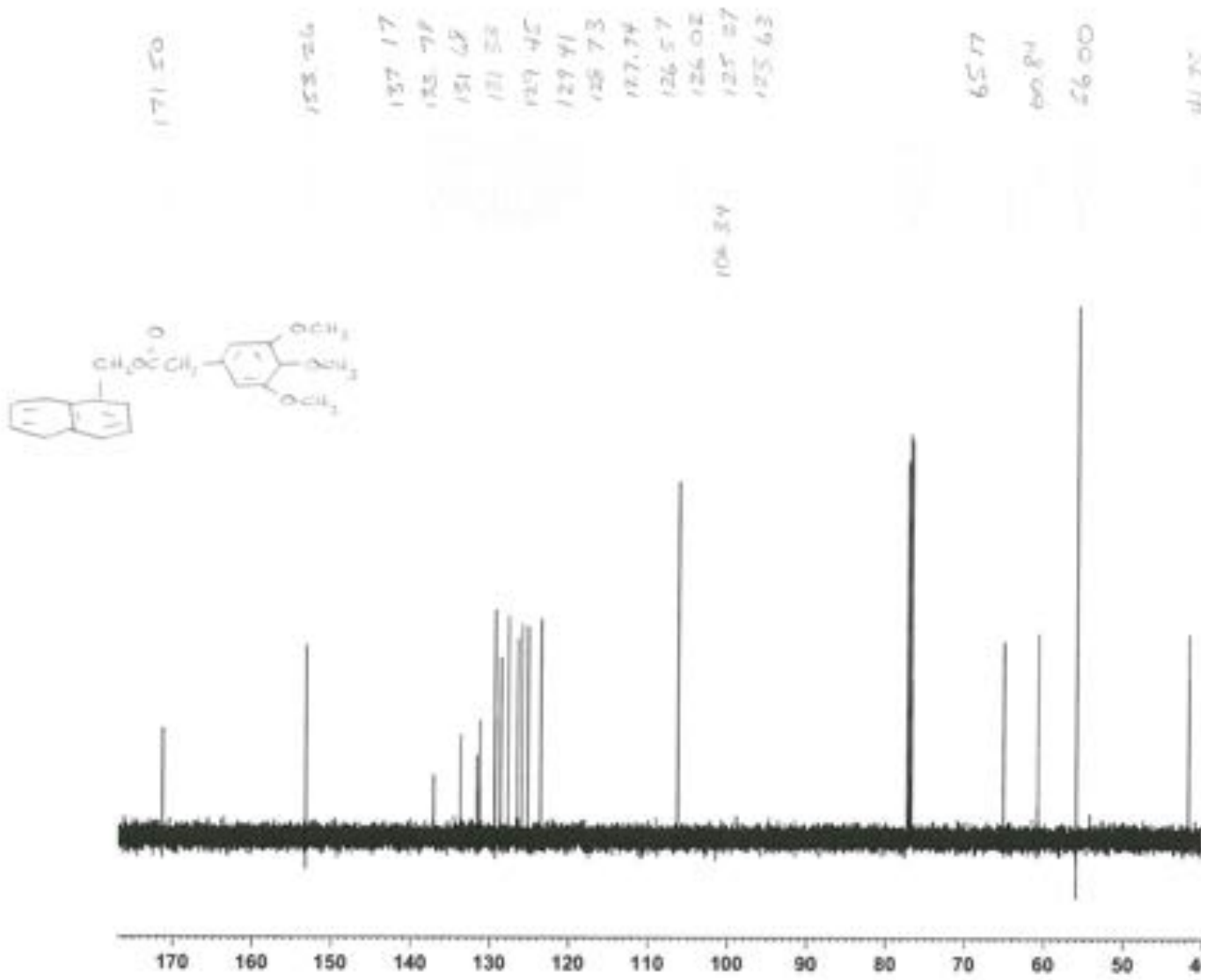


2-Naphthylmethyl 3,4,5-trimethoxyphenylacetate (1f)

${ }^{1} \mathrm{H}-\mathrm{NMR}\left(500 \mathrm{MHz}, \mathrm{CDCl}_{3}\right)$

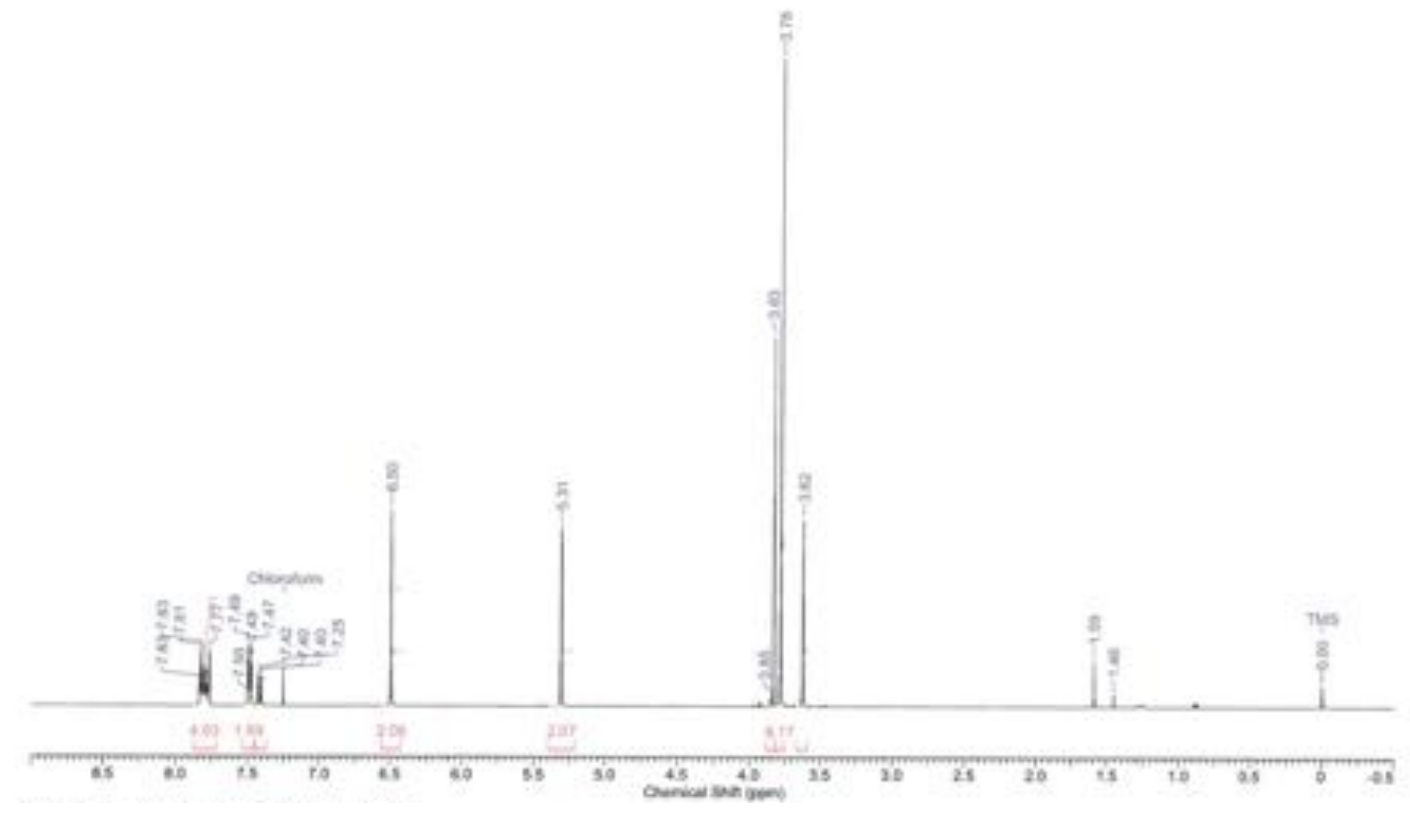

${ }^{13} \mathrm{C}$ UDEFT NMR (125 MHz, $\left.\mathrm{CDCl}_{3}\right)$

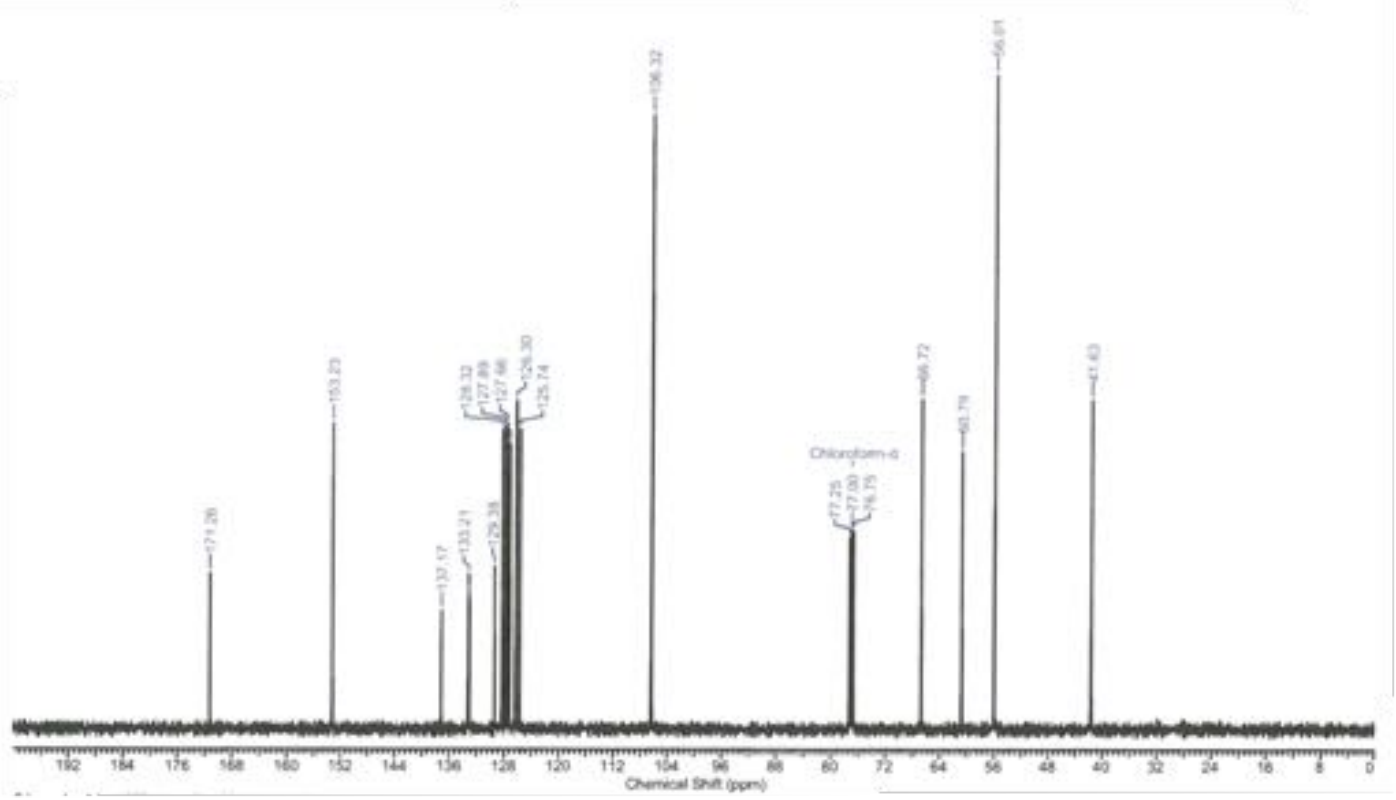




\section{4-Methyl-1-naphthylmethyl 1-naphthylacetate (1g)}

${ }^{1} \mathrm{H}-\mathrm{NMR}\left(500 \mathrm{MHz}^{\mathrm{CDCl}} 3\right.$ )

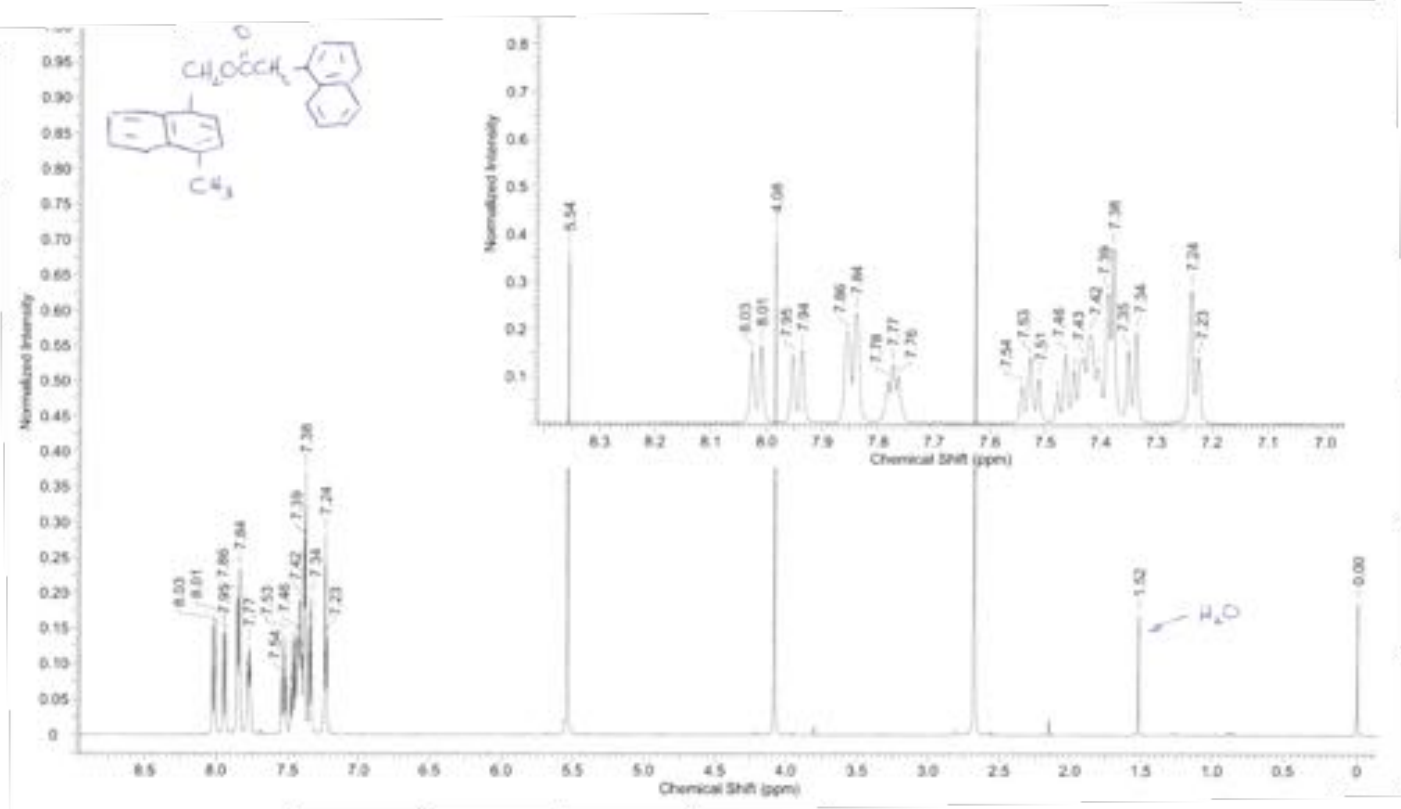

${ }^{13} \mathrm{C}$ UDEFT NMR (125 MHz, $\left.\mathrm{CDCl}_{3}\right)$

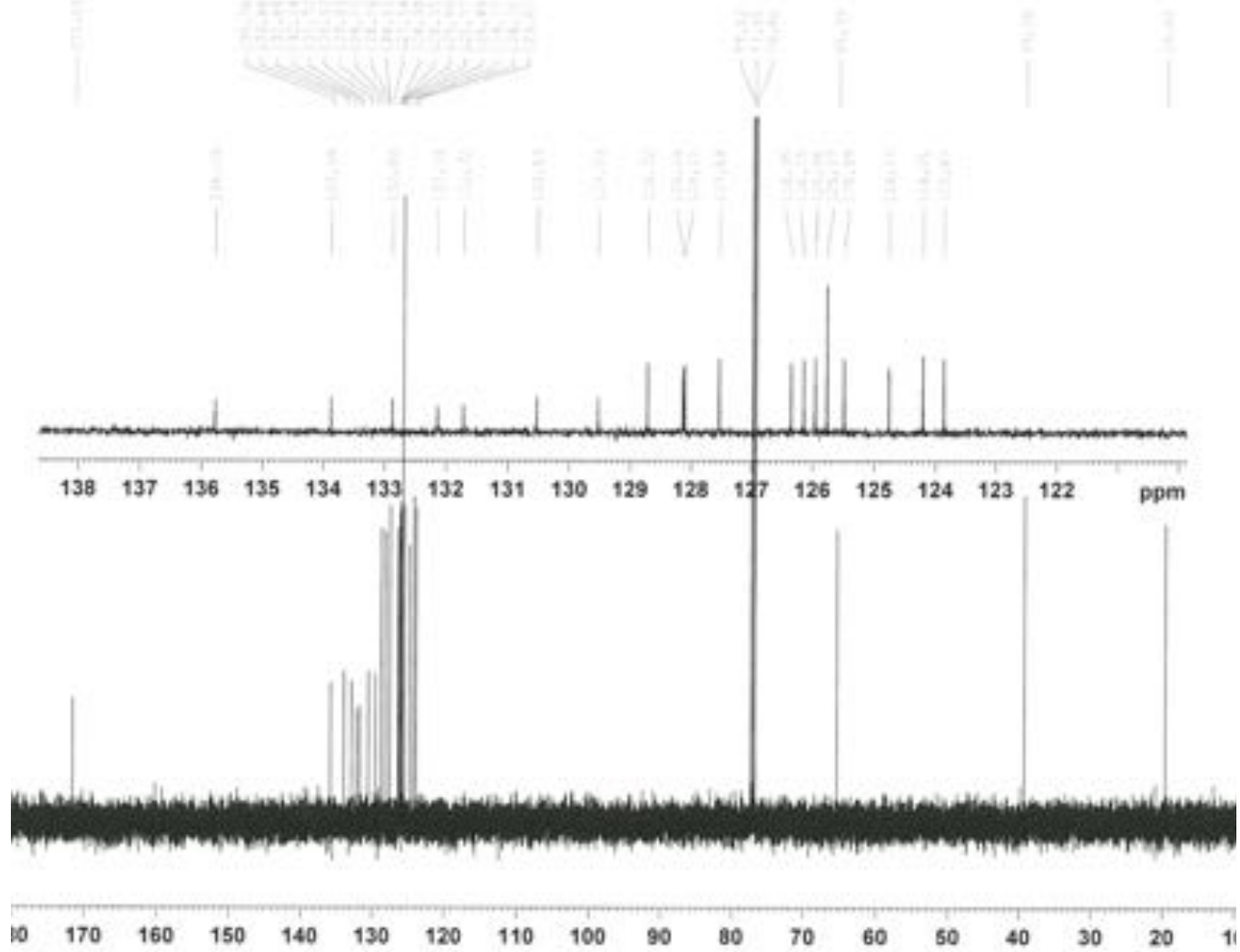


4-Cyano-1-naphthylmethyl 1-naphthylacetate (1h)

${ }^{1} \mathrm{H}-\mathrm{NMR}\left(500 \mathrm{MHz}, \mathrm{CDCl}_{3}\right)$

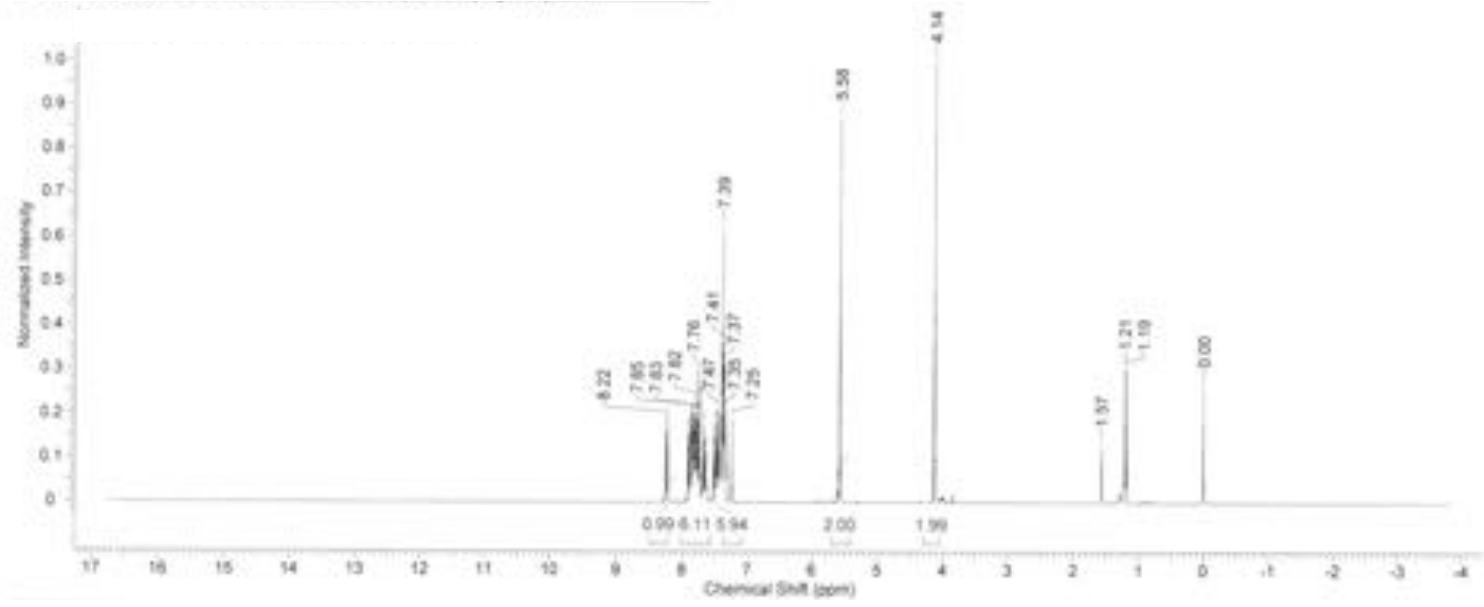

${ }^{13} \mathrm{C}$ UDEFT NMR (125 MHz, $\left.\mathrm{CDCl}_{3}\right)$
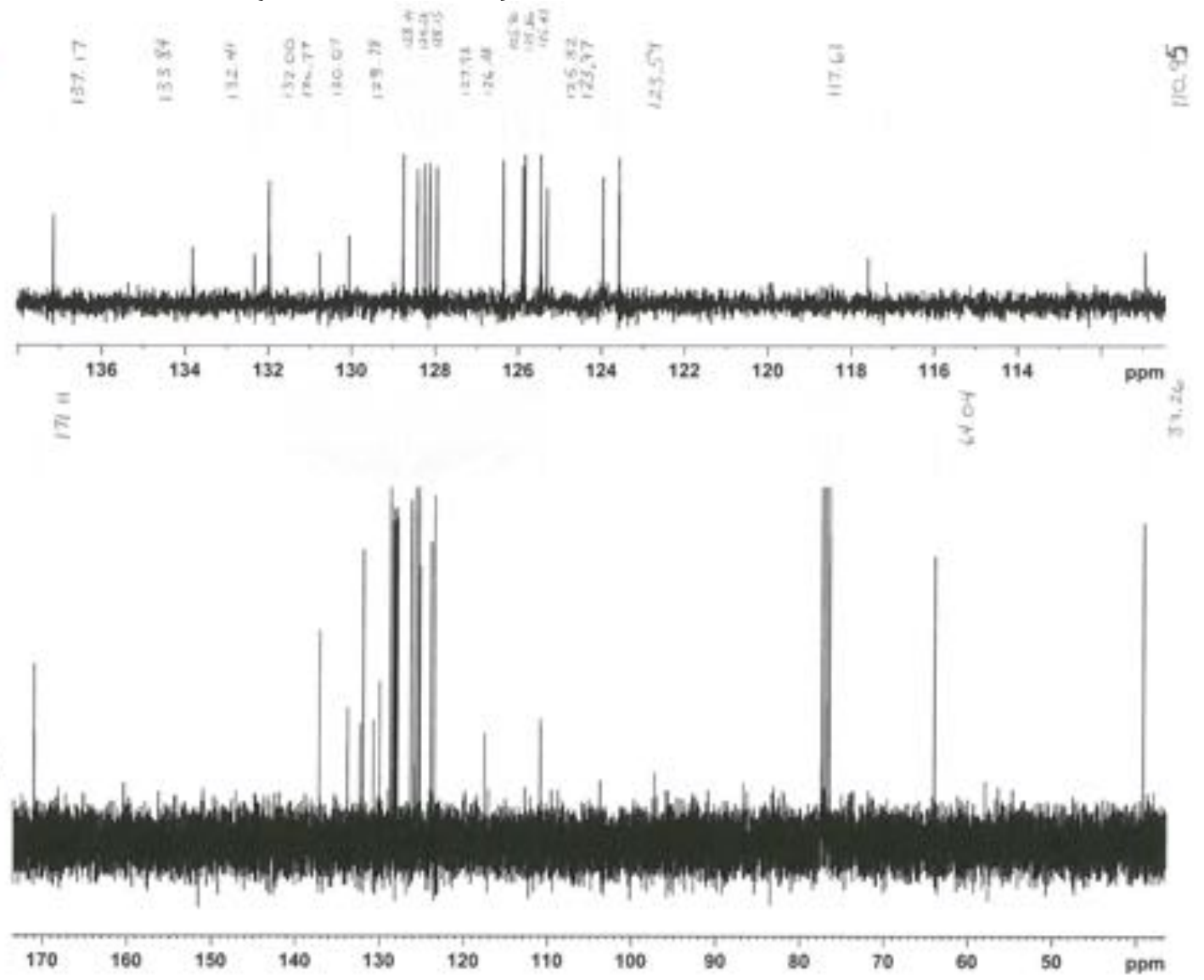
4-Cyano-1-naphthylmethyl 3,4,5-trimethoxyphenylacetate (1i)

${ }^{1} \mathrm{H}-\mathrm{NMR}\left(500 \mathrm{MHz}^{\mathrm{CDCl}} 3\right.$ )

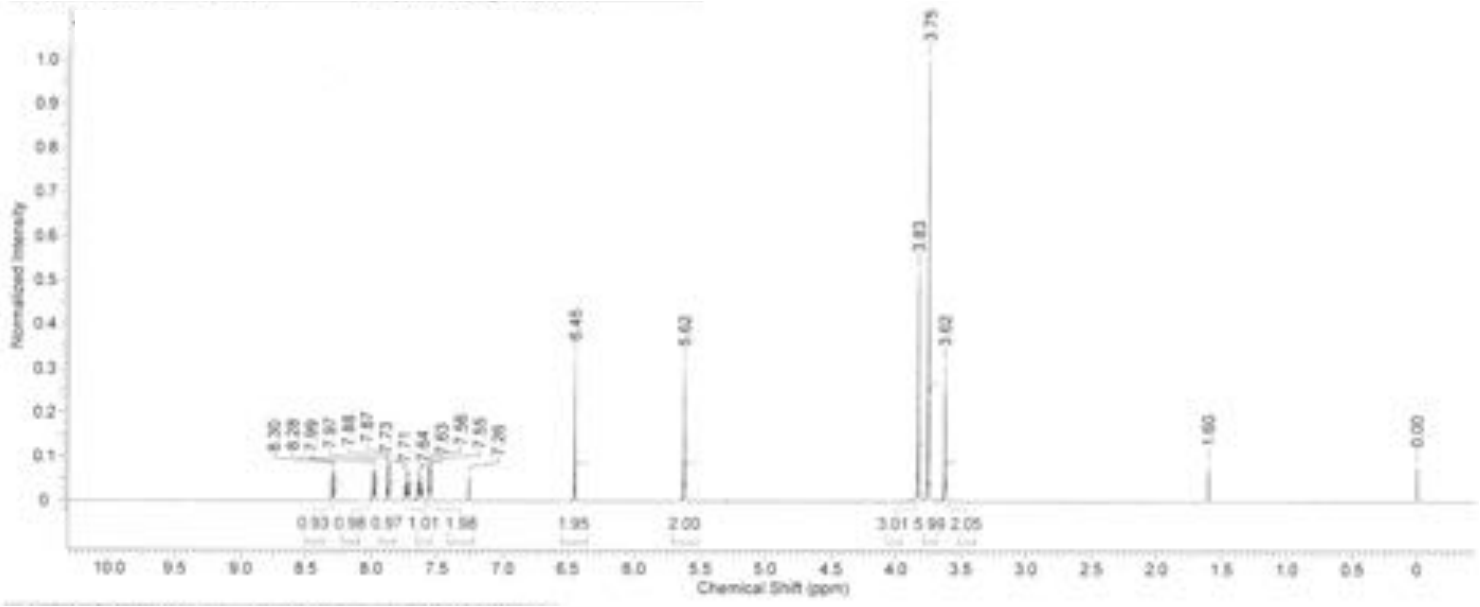

${ }^{13} \mathrm{C}$ UDEFT NMR (125 MHz, $\left.\mathrm{CDCl}_{3}\right)$
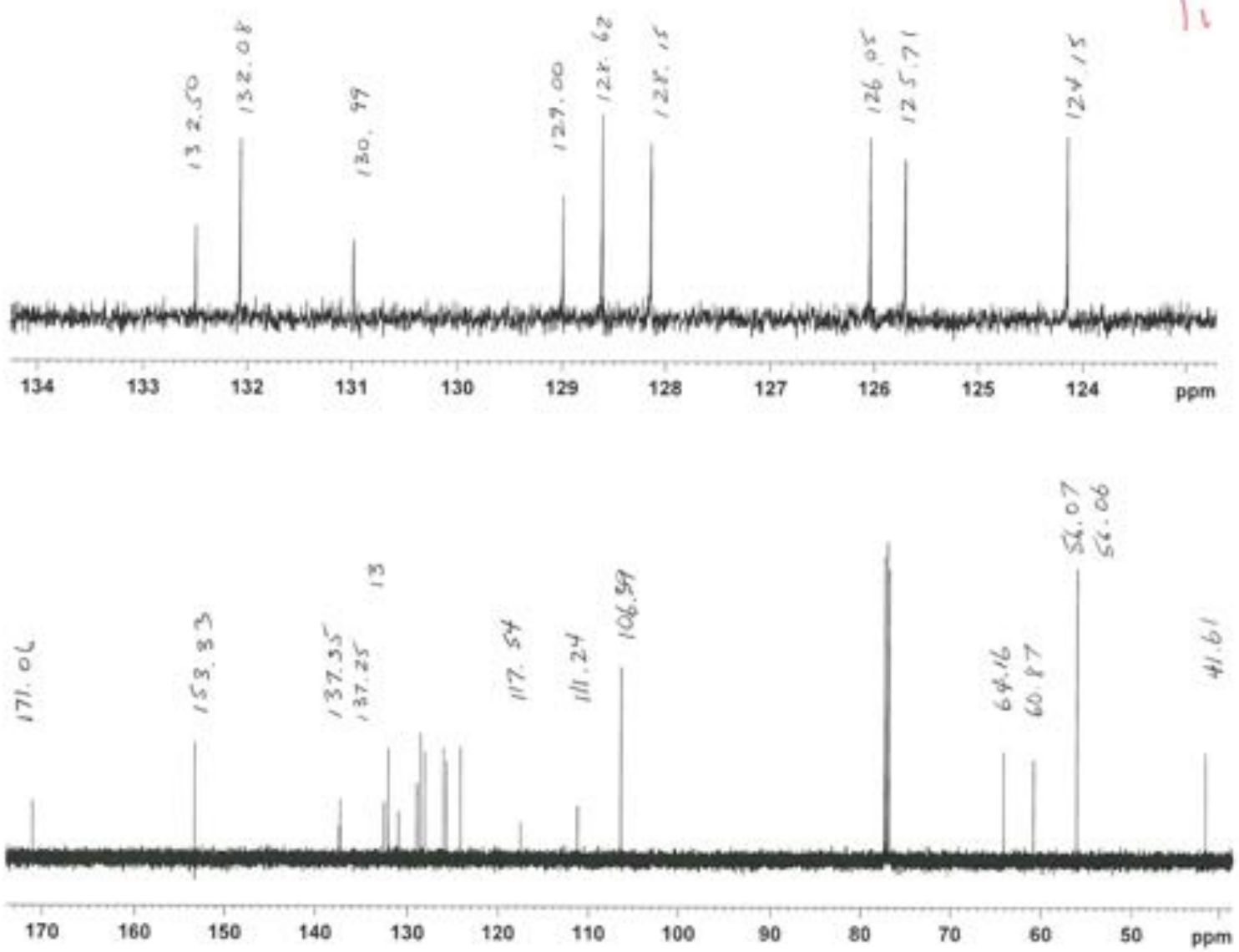
1-(1-Naphthyl)-2-(1-naphthyl)ethane (2a)

${ }^{1} \mathrm{H}-\mathrm{NMR}\left(500 \mathrm{MHz}^{\mathrm{CDCl}}{ }_{3}\right)$

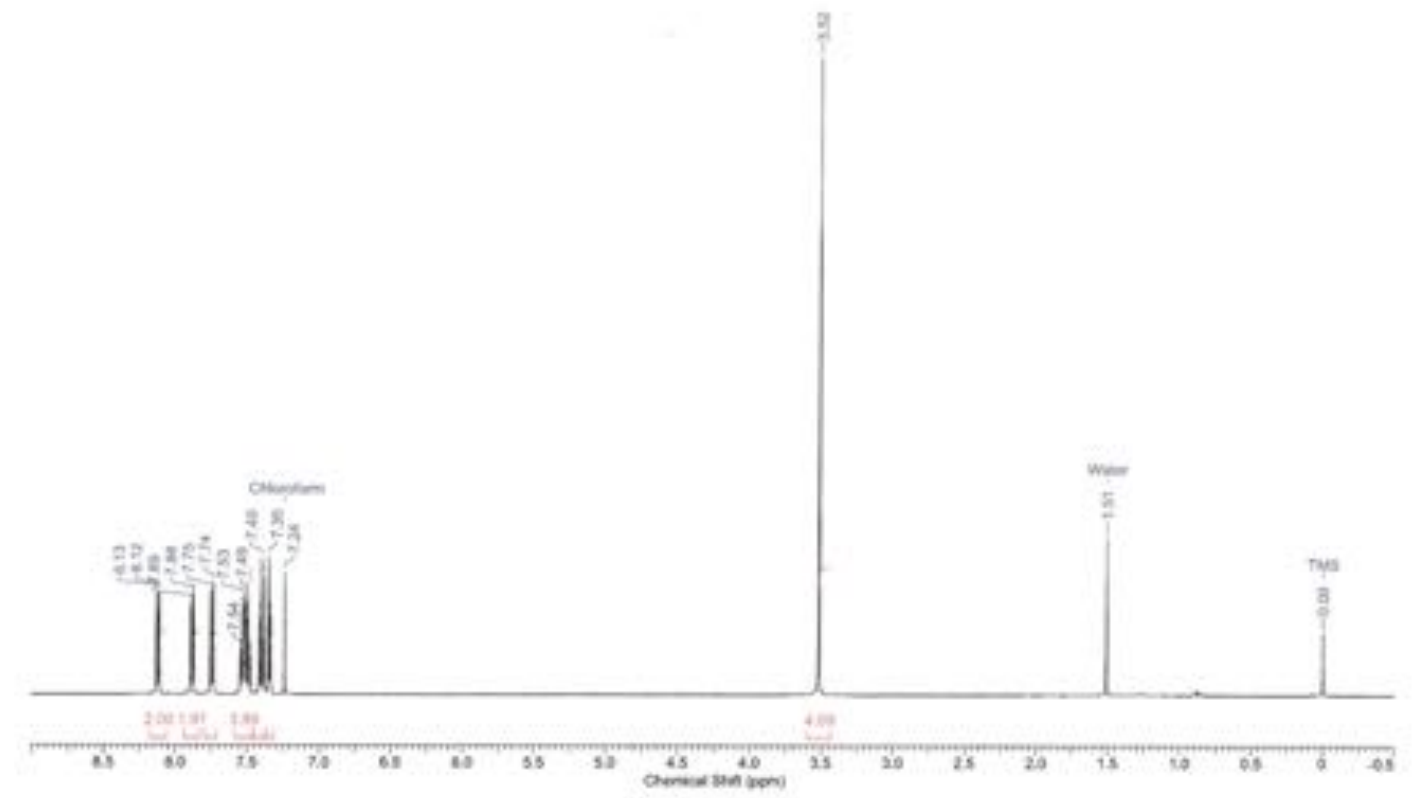

${ }^{13} \mathrm{C}$ UDEFT NMR (125 MHz, $\left.\mathrm{CDCl}_{3}\right)$

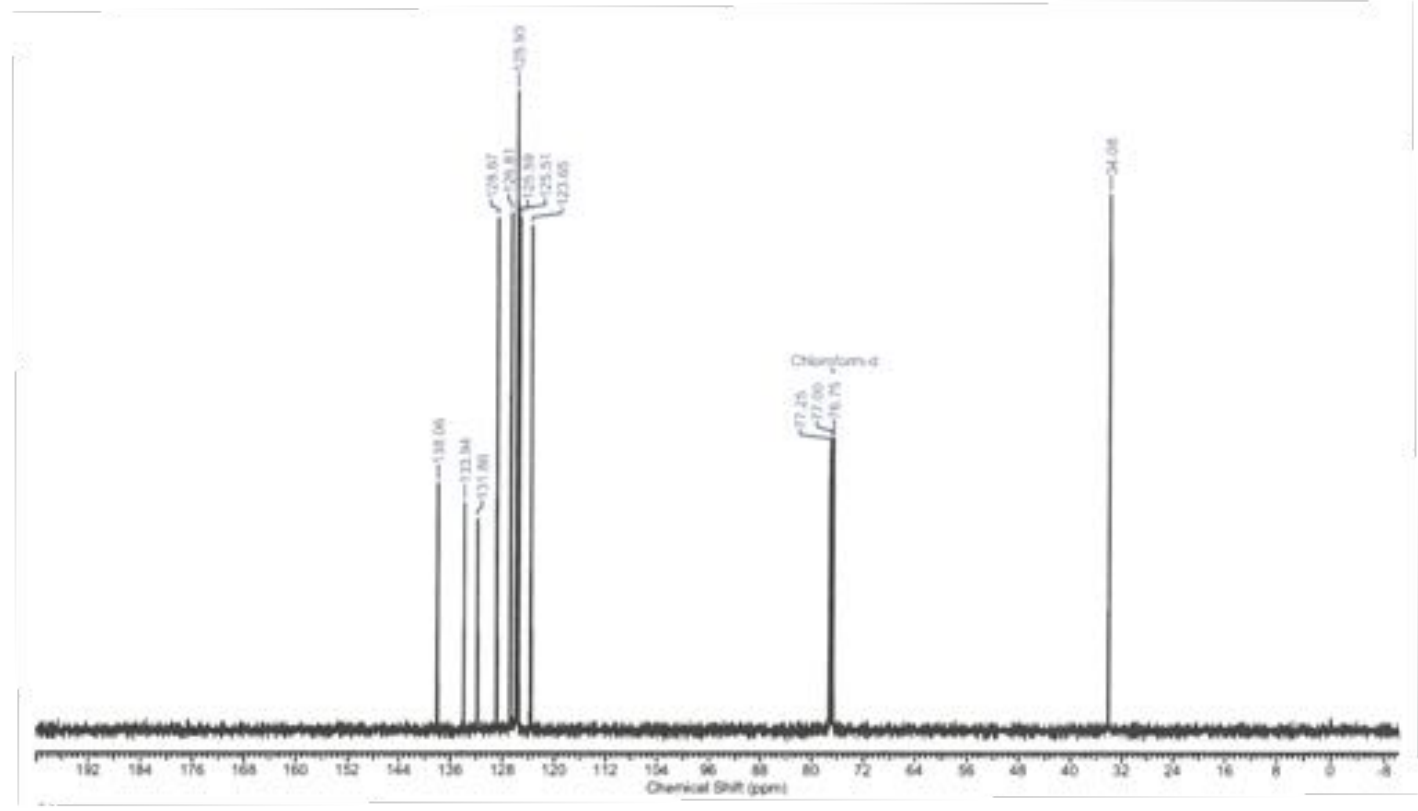


1-(1-Naphthyl)-2-(2-naphthyl)ethane (2b/c)

${ }^{1} \mathrm{H}-\mathrm{NMR}\left(500 \mathrm{MHz}, \mathrm{CDCl}_{3}\right)$

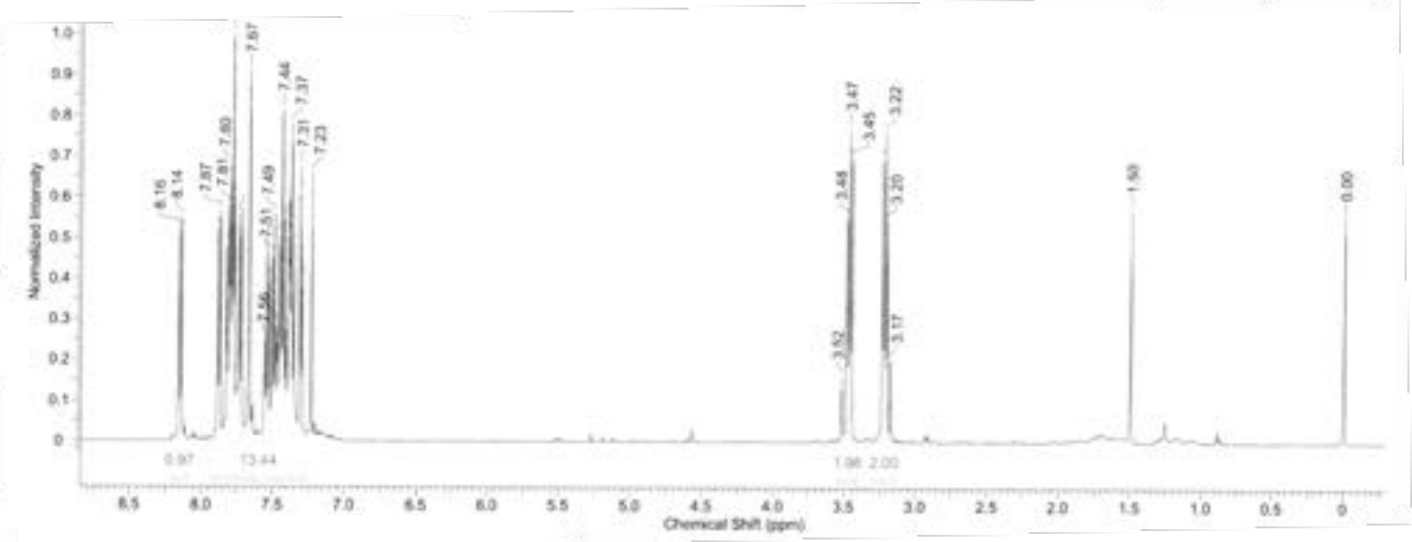

${ }^{13} \mathrm{C}$ UDEFT NMR (125 MHz, $\left.\mathrm{CDCl}_{3}\right)$
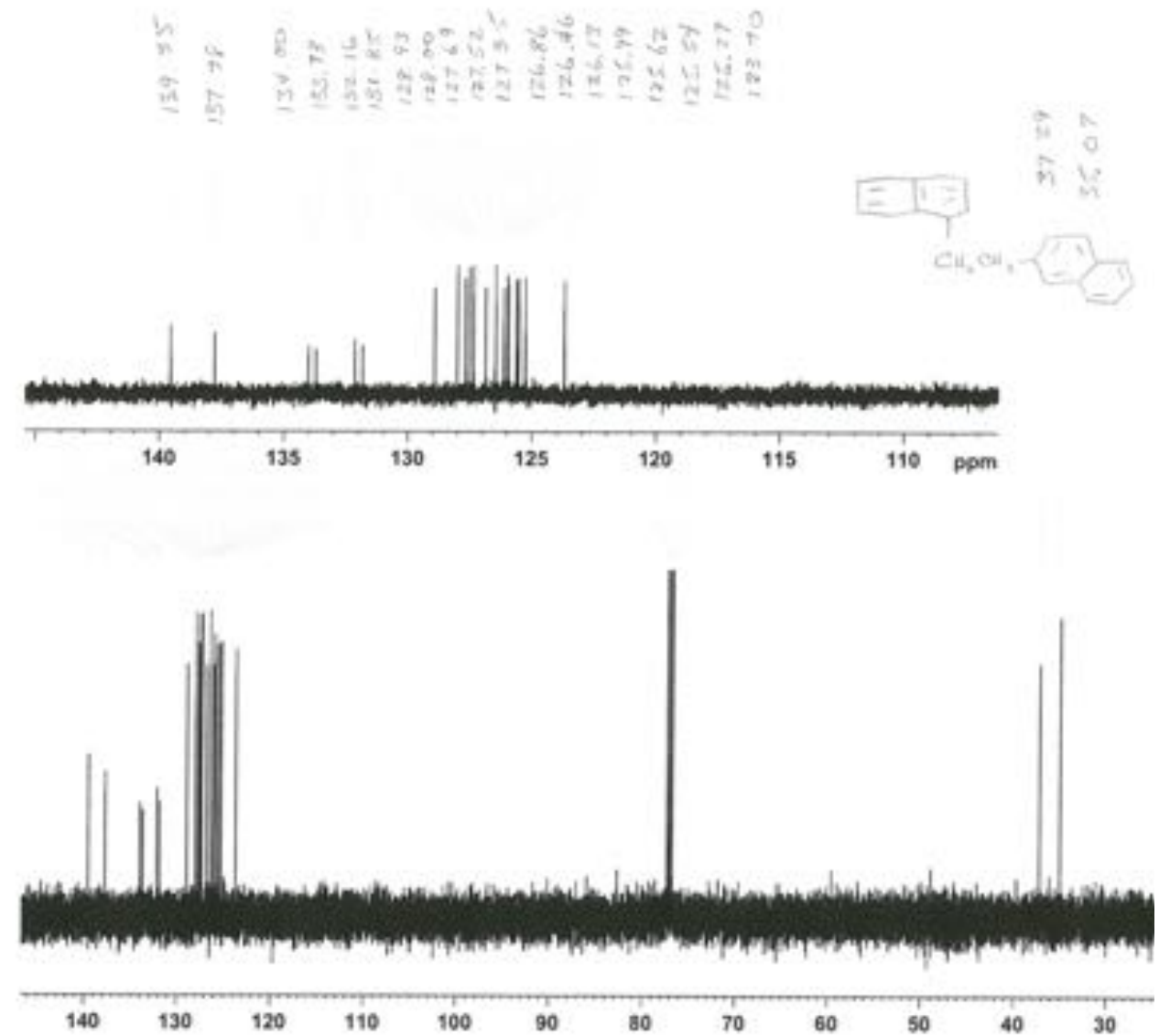
1-(2-Naphthyl)-2-(2-naphthyl)ethane (2d)

${ }^{1} \mathrm{H}-\mathrm{NMR}\left(500 \mathrm{MHz}, \mathrm{CDCl}_{3}\right)$

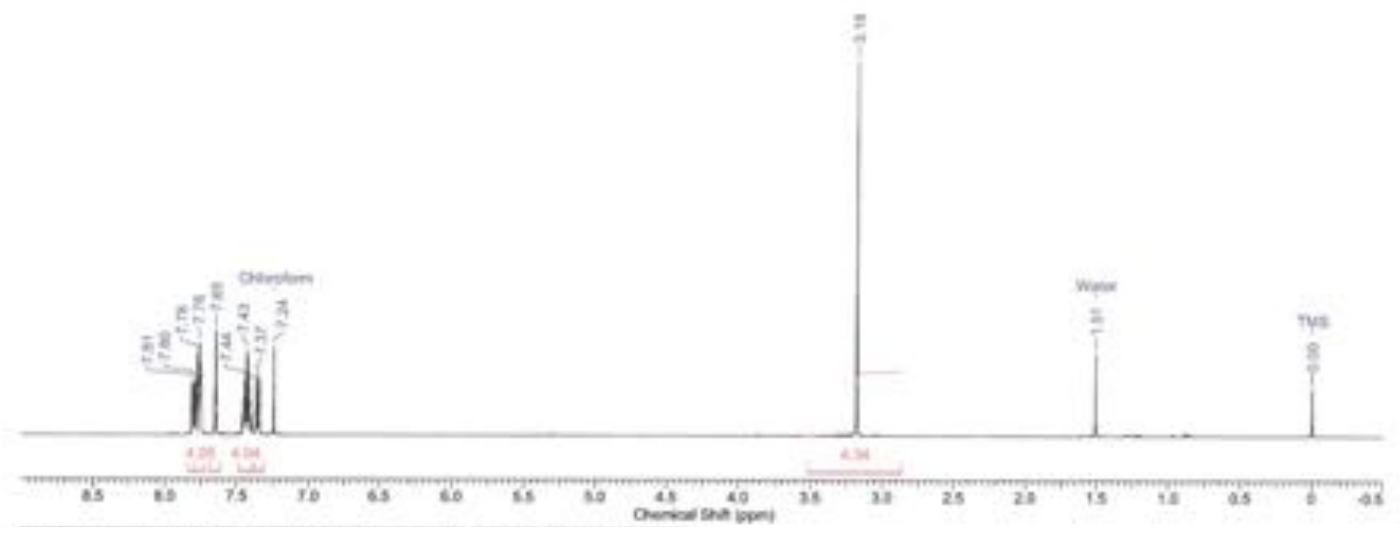

${ }^{13} \mathrm{C}$ UDEFT NMR (125 MHz, $\left.\mathrm{CDCl}_{3}\right)$
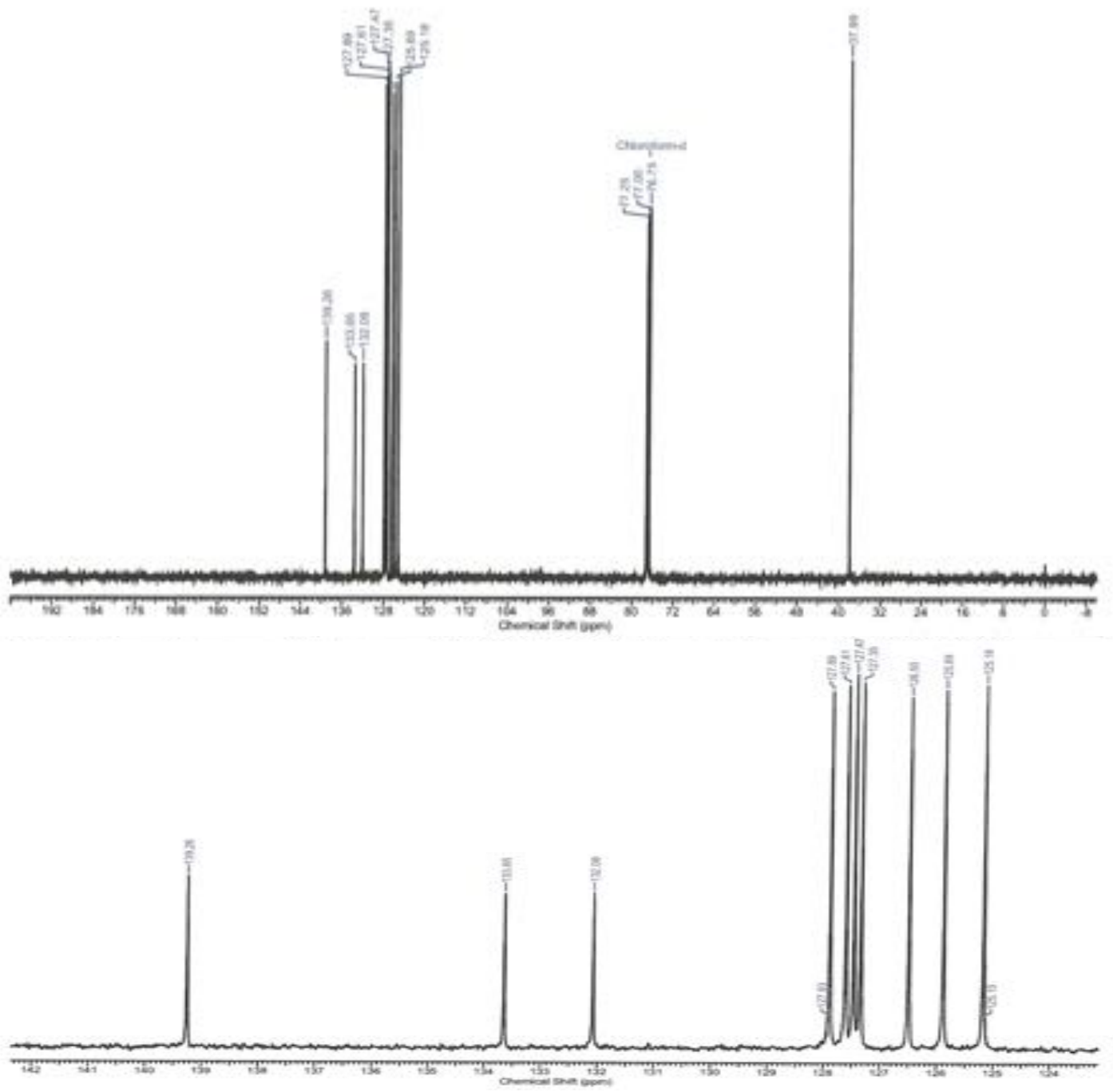
1-(1-Naphthyl)-2-(3,4,5-trimethoxyphenyl)ethane (2e)

${ }^{1} \mathrm{H}-\mathrm{NMR}\left(500 \mathrm{MHz}, \mathrm{CDCl}_{3}\right)$

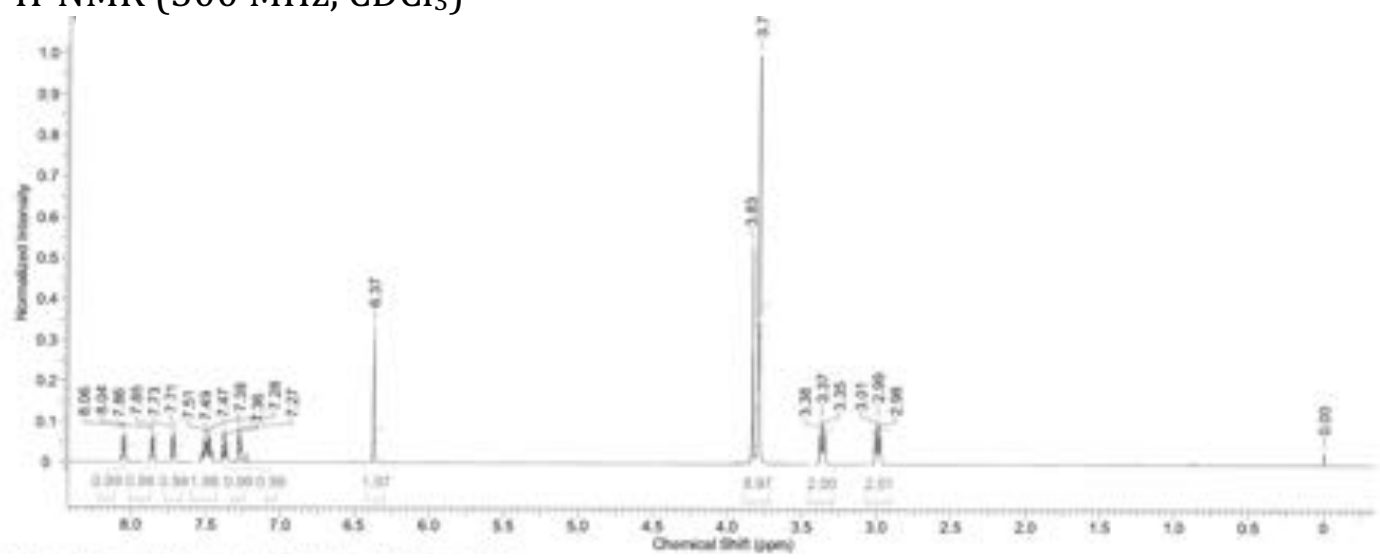

${ }^{13} \mathrm{C}$ UDEFT NMR (125 MHz, $\left.\mathrm{CDCl}_{3}\right)$
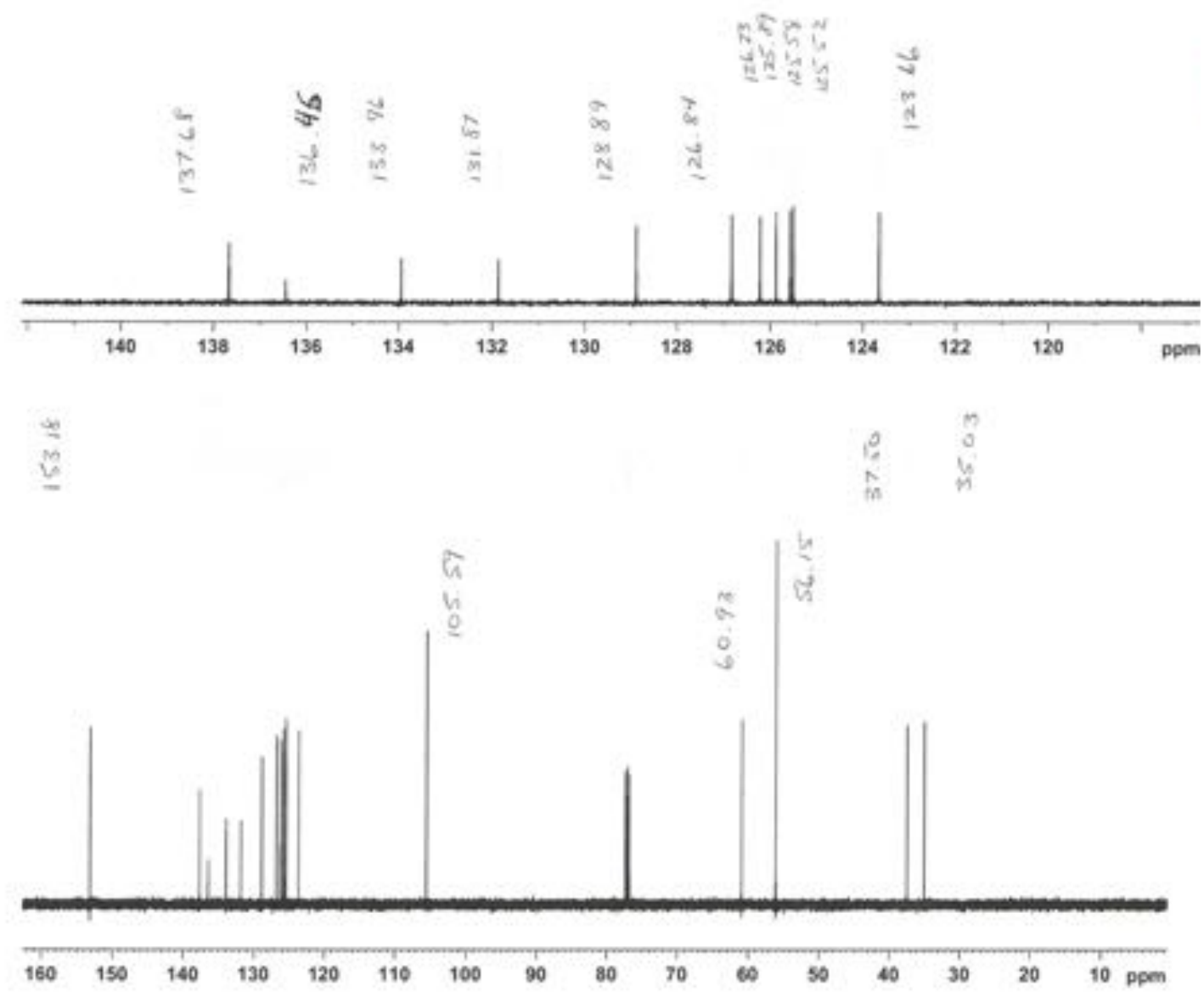
1-(2-Naphthyl)-2-(3,4,5-trimethoxyphenyl)ethane (2f)

${ }^{1} \mathrm{H}-\mathrm{NMR}\left(500 \mathrm{MHz}, \mathrm{CDCl}_{3}\right.$ )

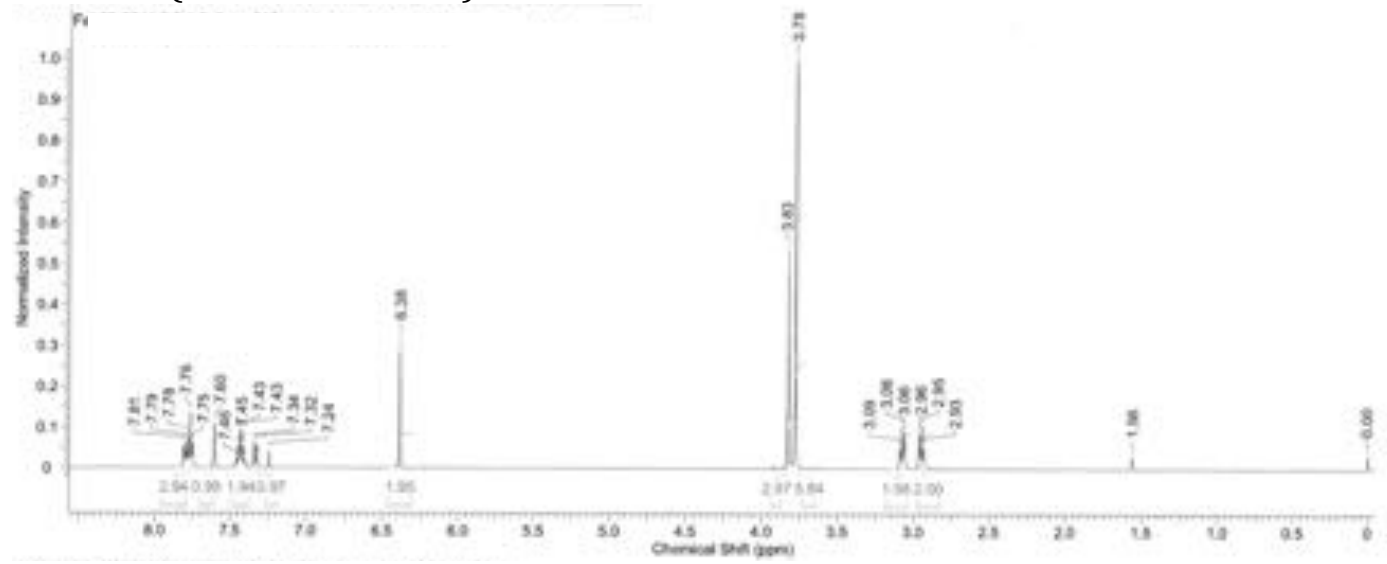

${ }^{13} \mathrm{C}$ UDEFT NMR (125 MHz, $\left.\mathrm{CDCl}_{3}\right)$
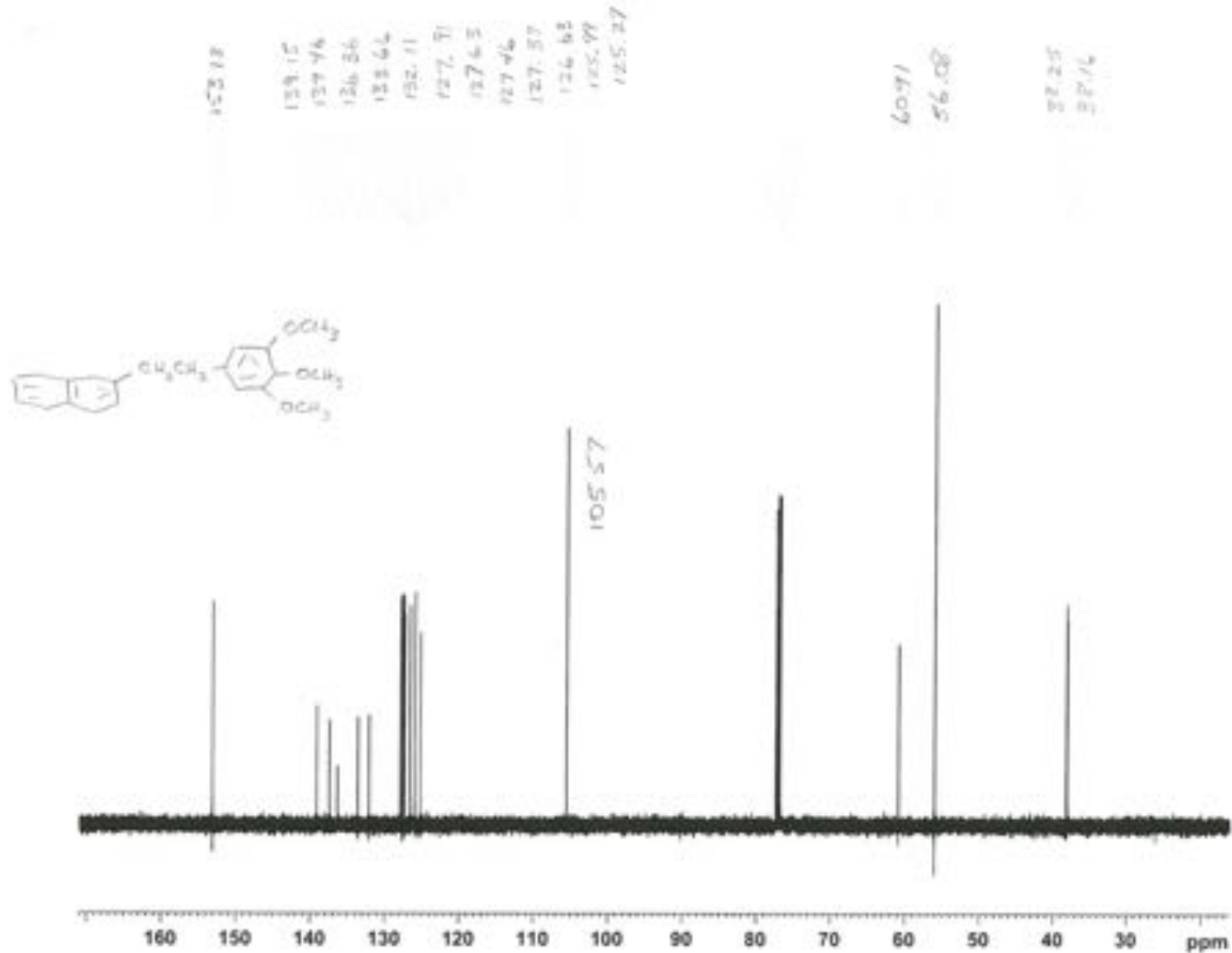
1-(4-Methyl-1-naphthyl)-2-(1-naphthyl)ethane (2g)

${ }^{1} \mathrm{H}-\mathrm{NMR}\left(500 \mathrm{MHz}, \mathrm{CDCl}_{3}\right)$

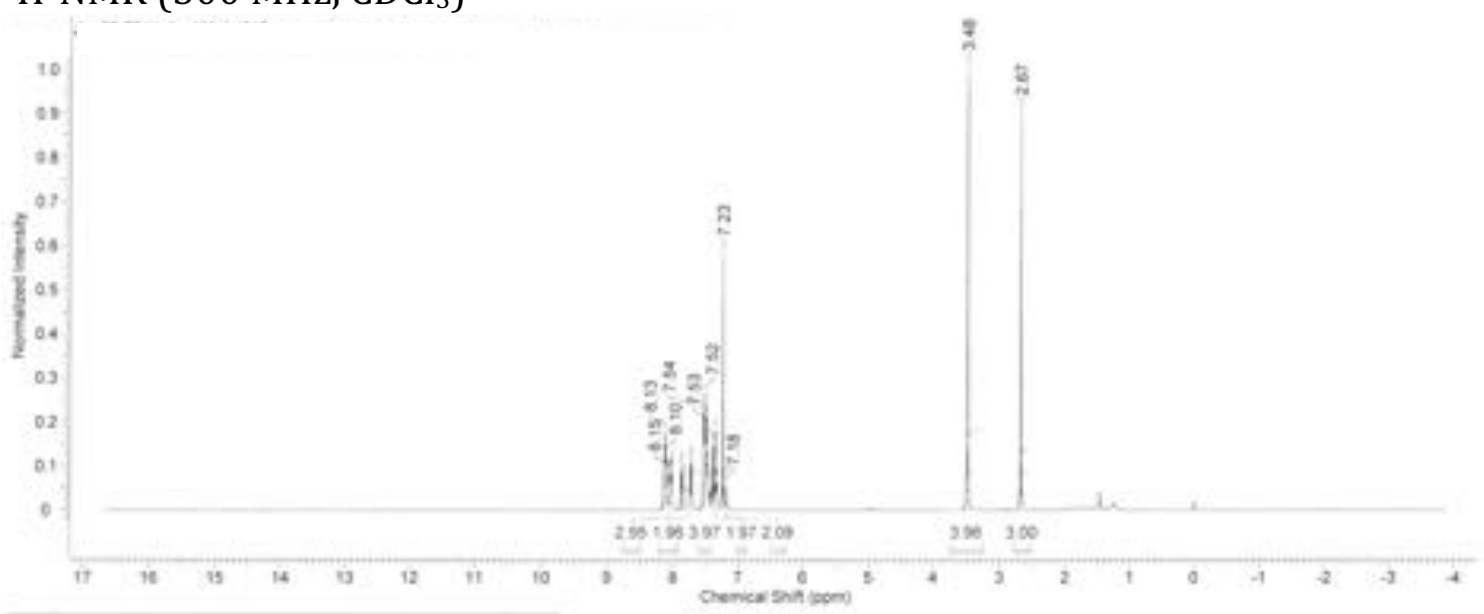

${ }^{13} \mathrm{C}$ UDEFT NMR (125 MHz, $\left.\mathrm{CDCl}_{3}\right)$

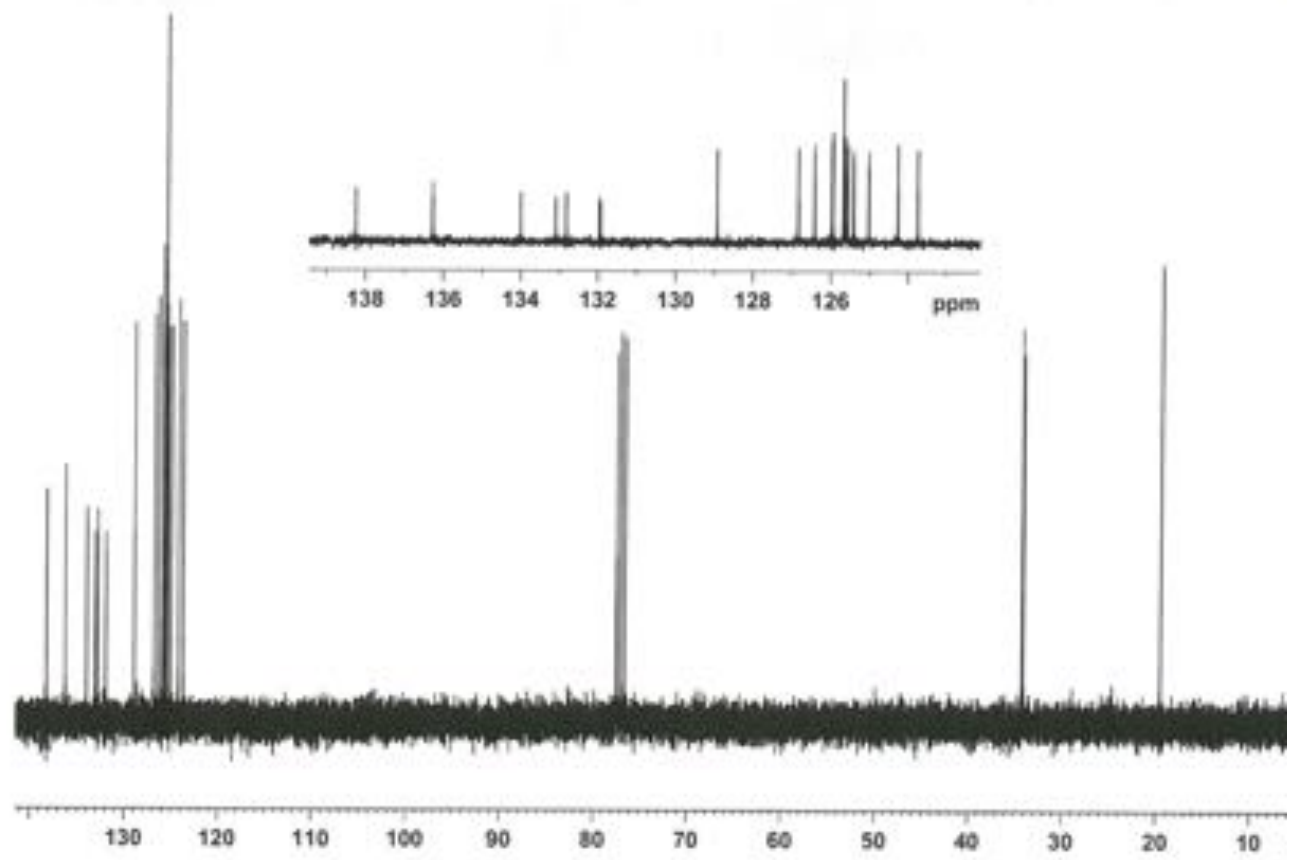


1-(4-Cyano-1-naphthyl)-2-(1-naphthyl)ethane (2h)

${ }^{1} \mathrm{H}-\mathrm{NMR}\left(500 \mathrm{MHz}, \mathrm{CDCl}_{3}\right)$

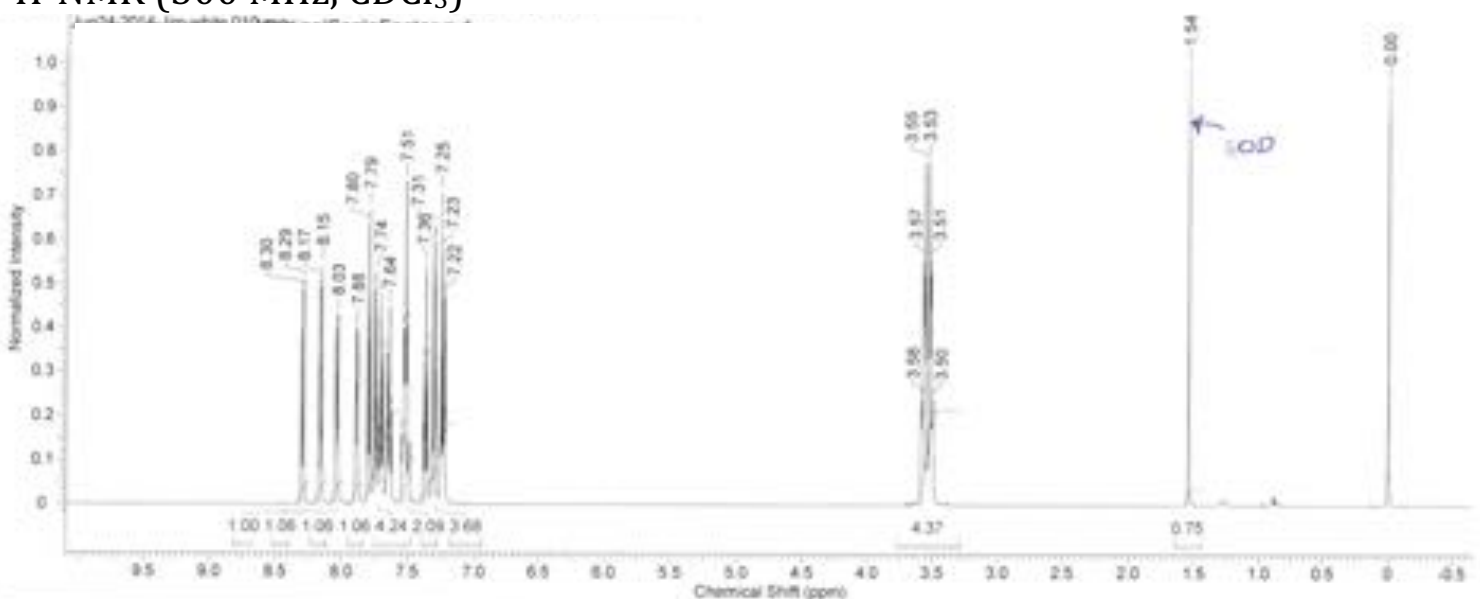

${ }^{13} \mathrm{C}$ UDEFT NMR (125 MHz, $\left.\mathrm{CDCl}_{3}\right)$
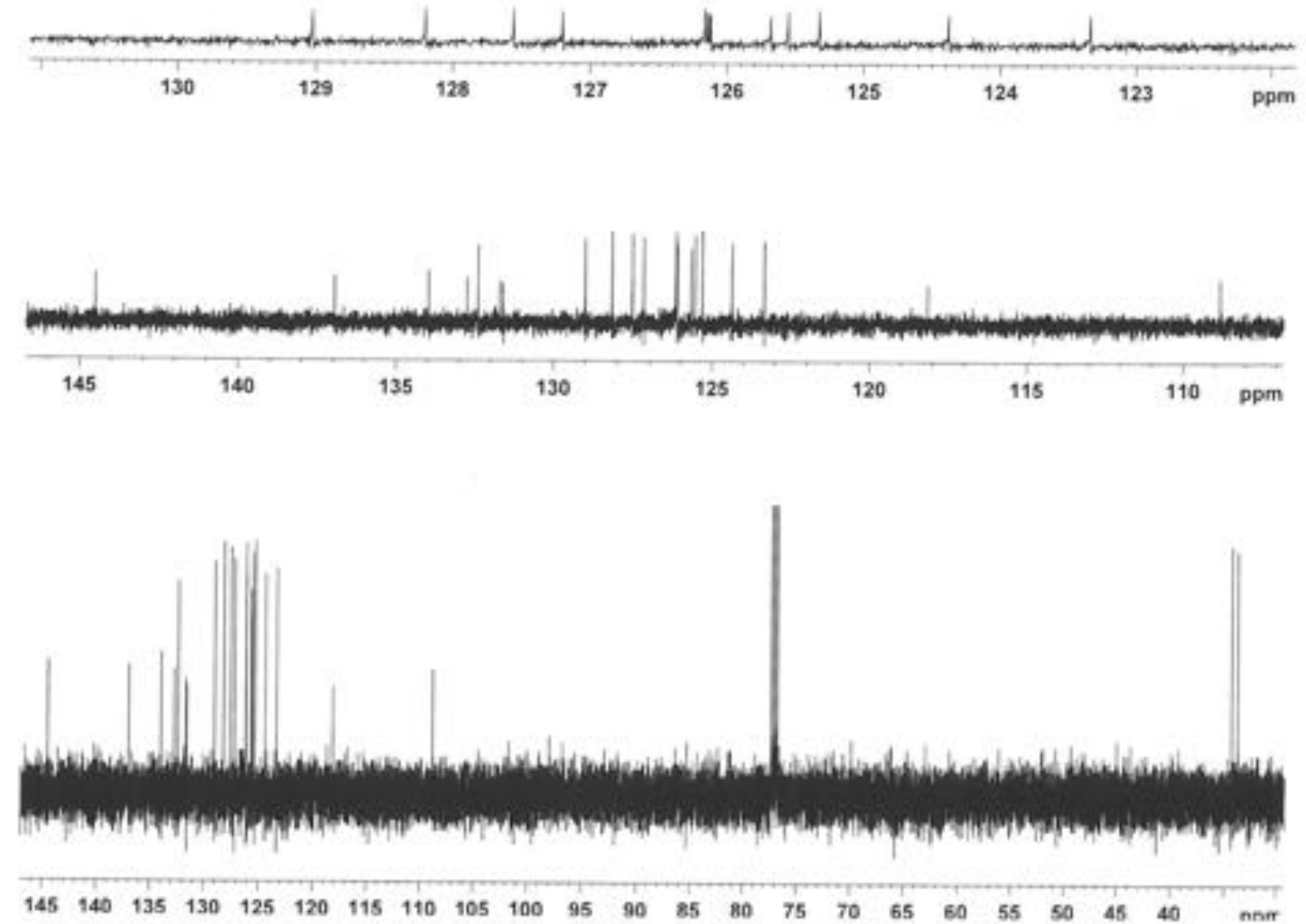
1-(4-Cyano-1-naphthyl)-2-(3,4,5-trimethoxyphenyl)ethane (2i)

${ }^{1} \mathrm{H}-\mathrm{NMR}\left(500 \mathrm{MHz}, \mathrm{CDCl}_{3}\right)$

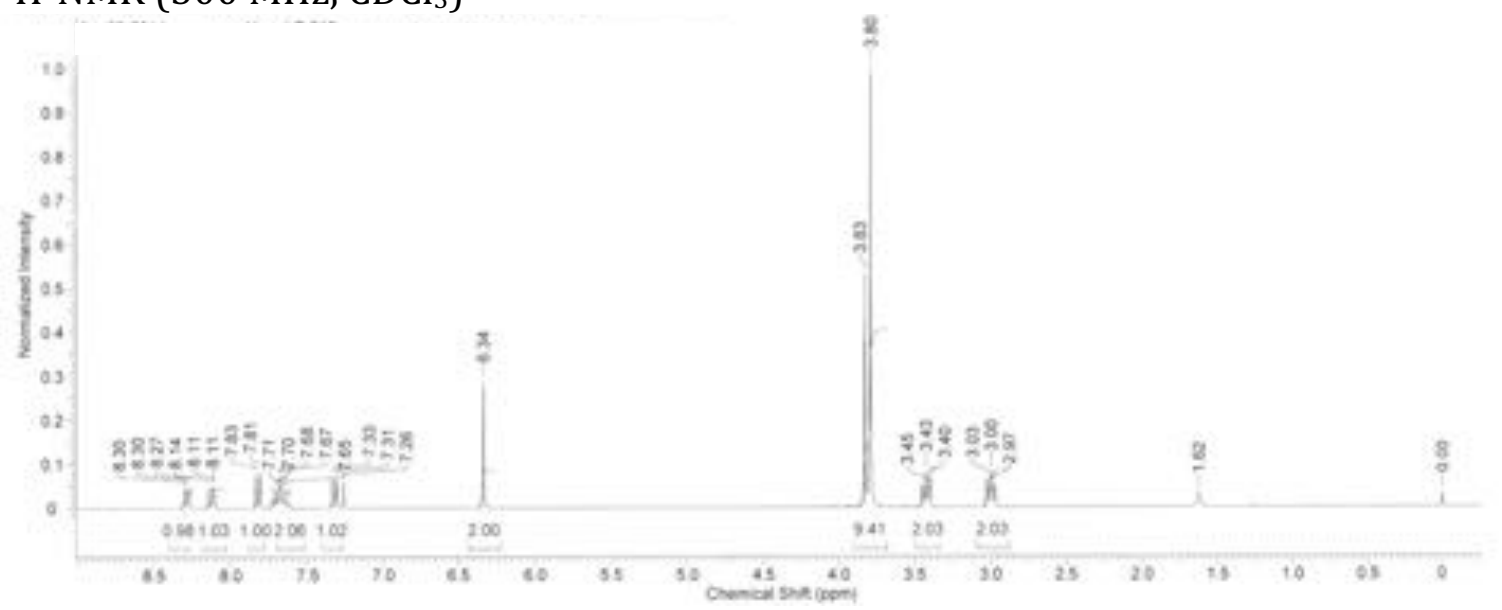

${ }^{13} \mathrm{C}$ UDEFT NMR (125 MHz, $\left.\mathrm{CDCl}_{3}\right)$
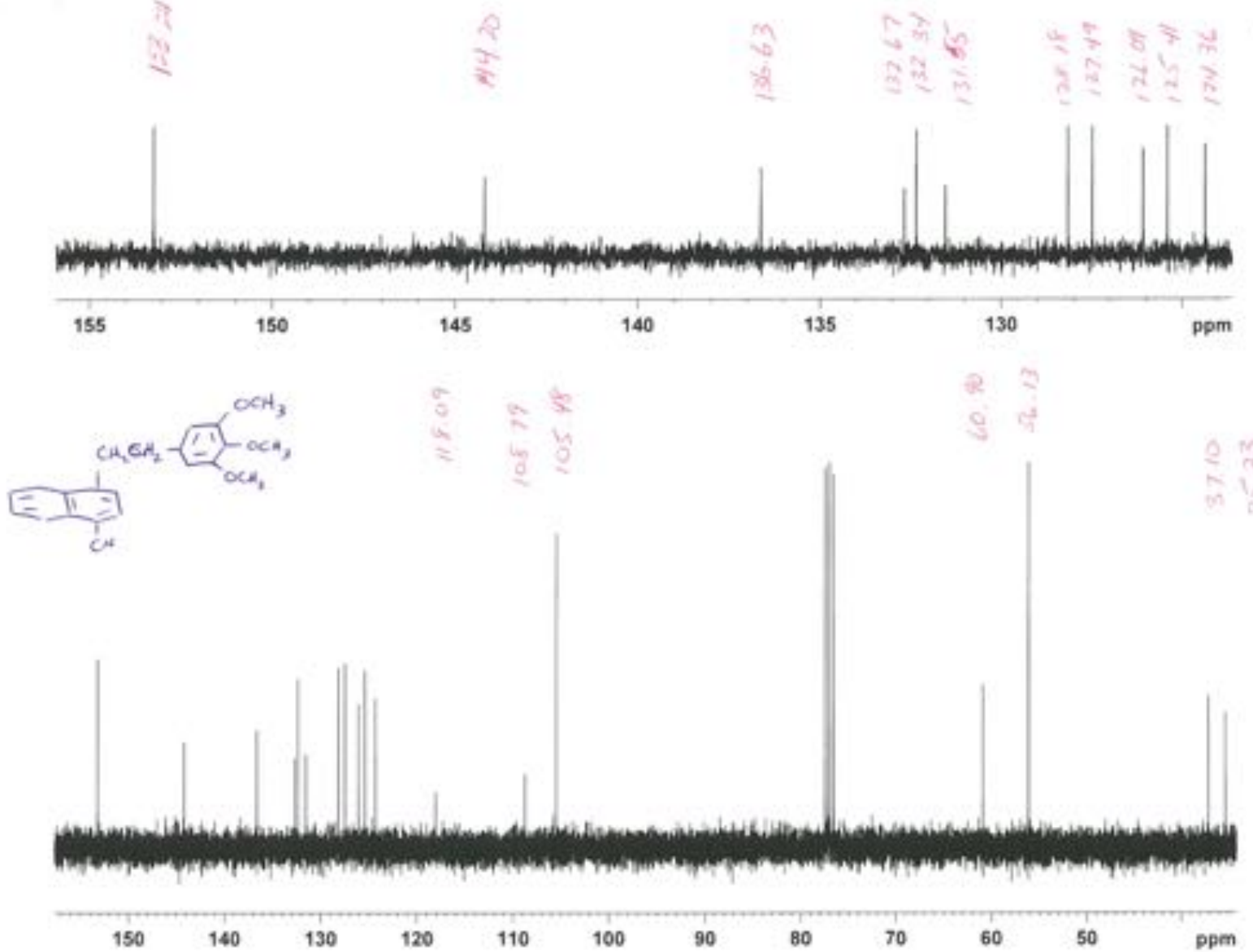


\section{1-Naphthylmethyl methylether (3)}

${ }^{1} \mathrm{H}-\mathrm{NMR}\left(500 \mathrm{MHz}^{\mathrm{CDCl}}{ }_{3}\right.$ )

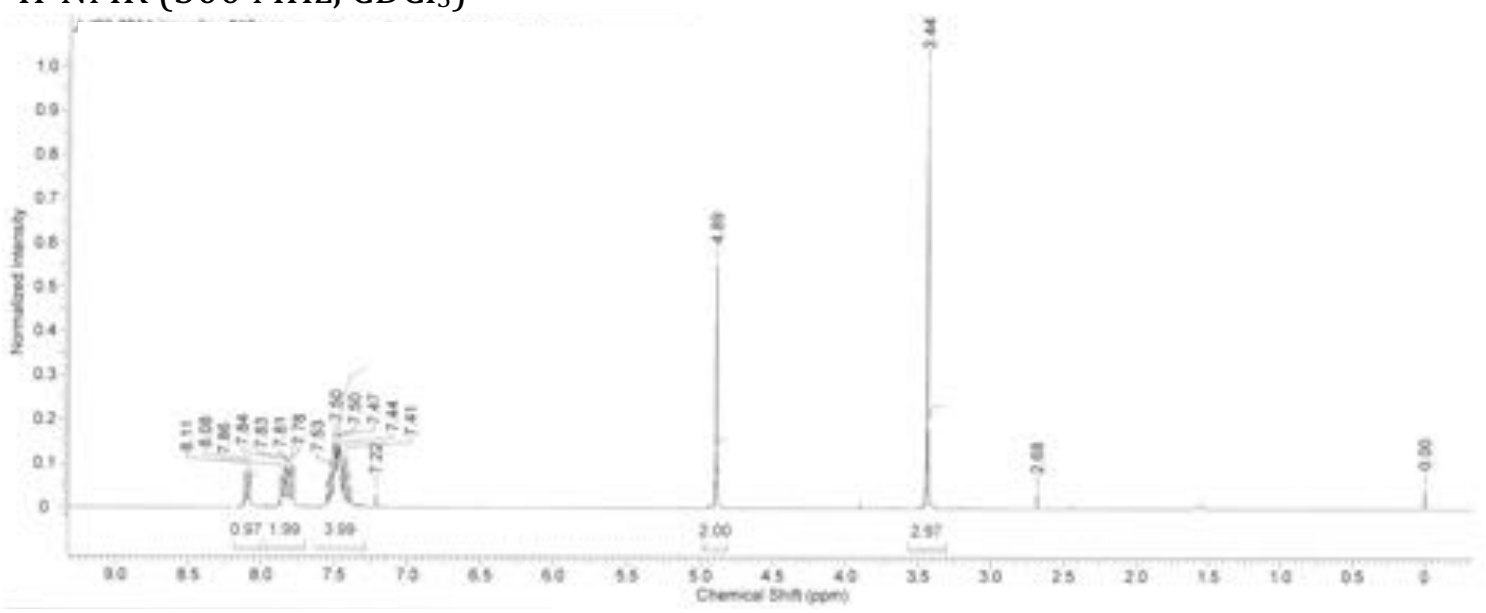

${ }^{13} \mathrm{C}$ UDEFT NMR (125 MHz, $\mathrm{CDCl}_{3}$ )
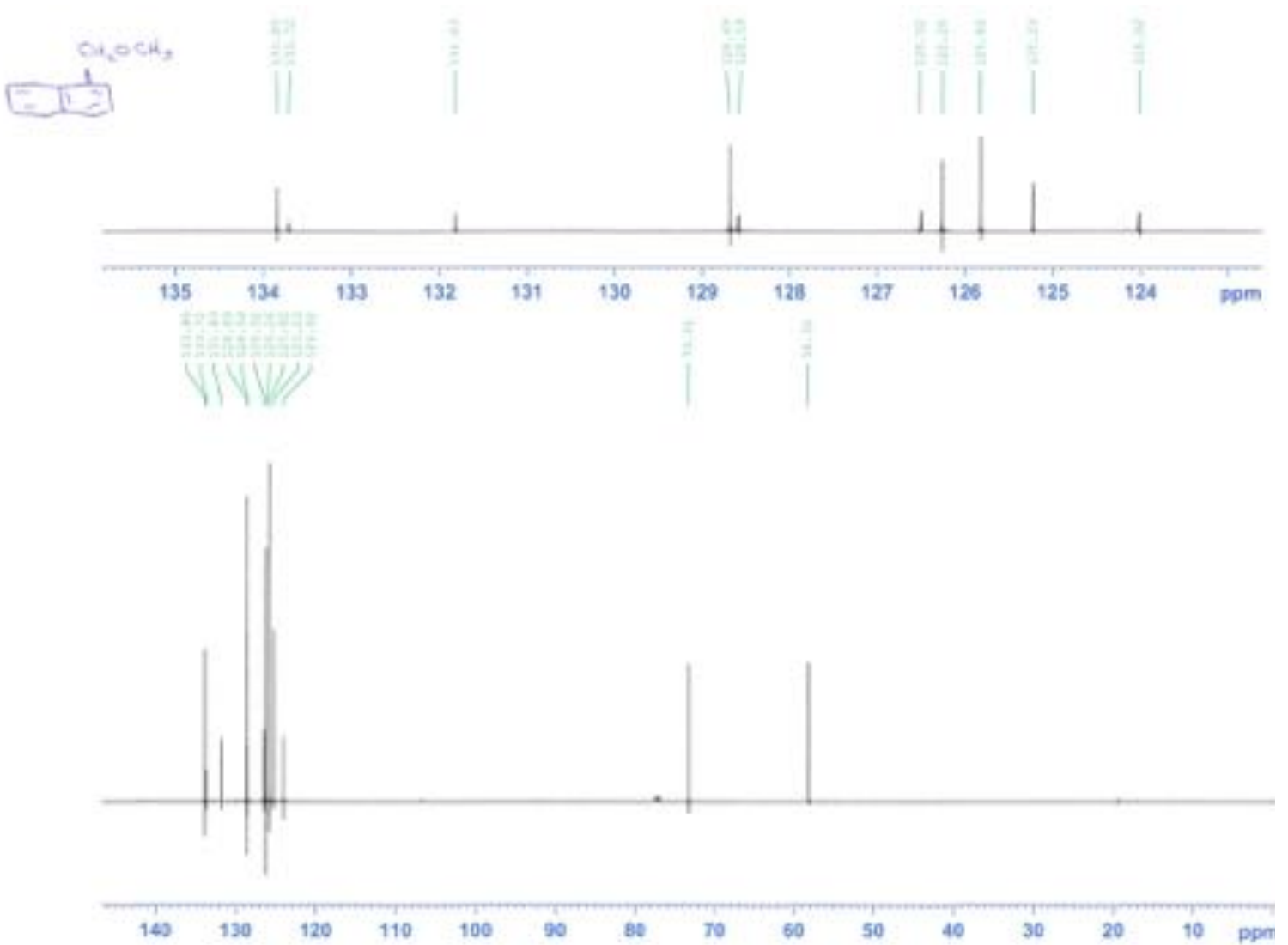
2-Naphthylmethyl methylether (4)

${ }^{1} \mathrm{H}-\mathrm{NMR}$ (500 MHz, $\mathrm{CDCl}_{3}$ )

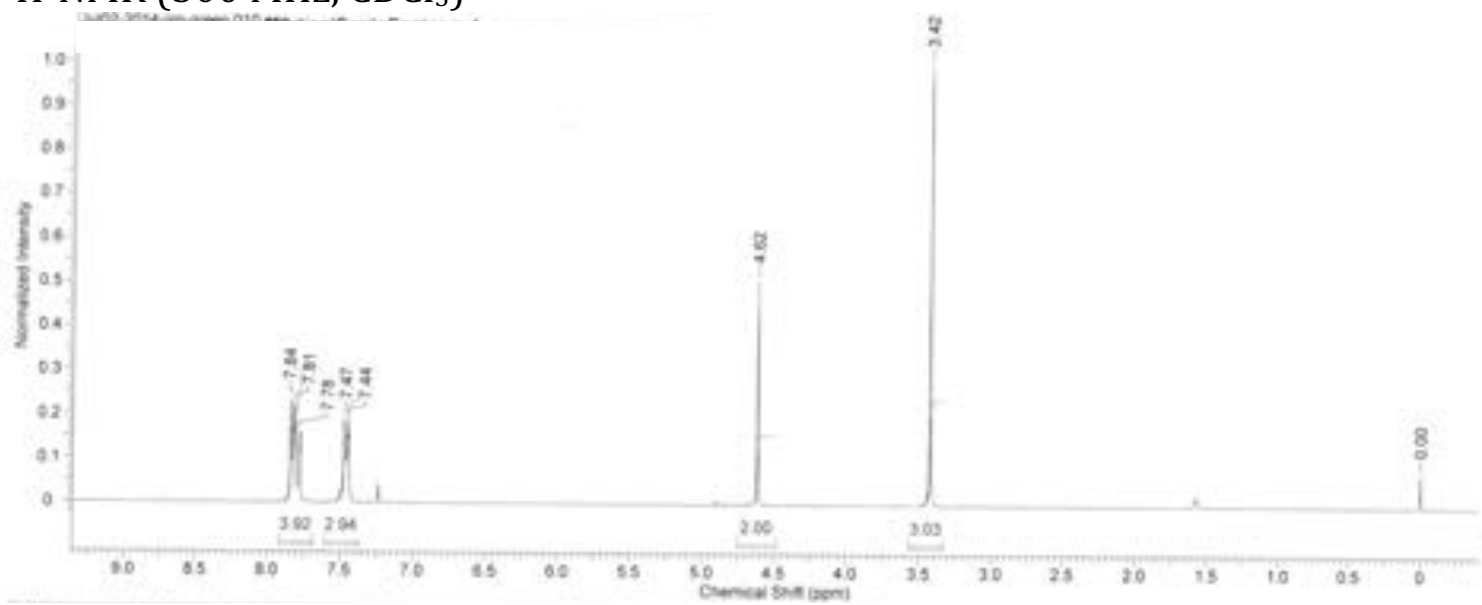




\section{4-Methyl-1-methoxymethylnaphthalene (5)}

${ }^{1} \mathrm{H}-\mathrm{NMR}$ (500 MHz, $\mathrm{CDCl}_{3}$ )

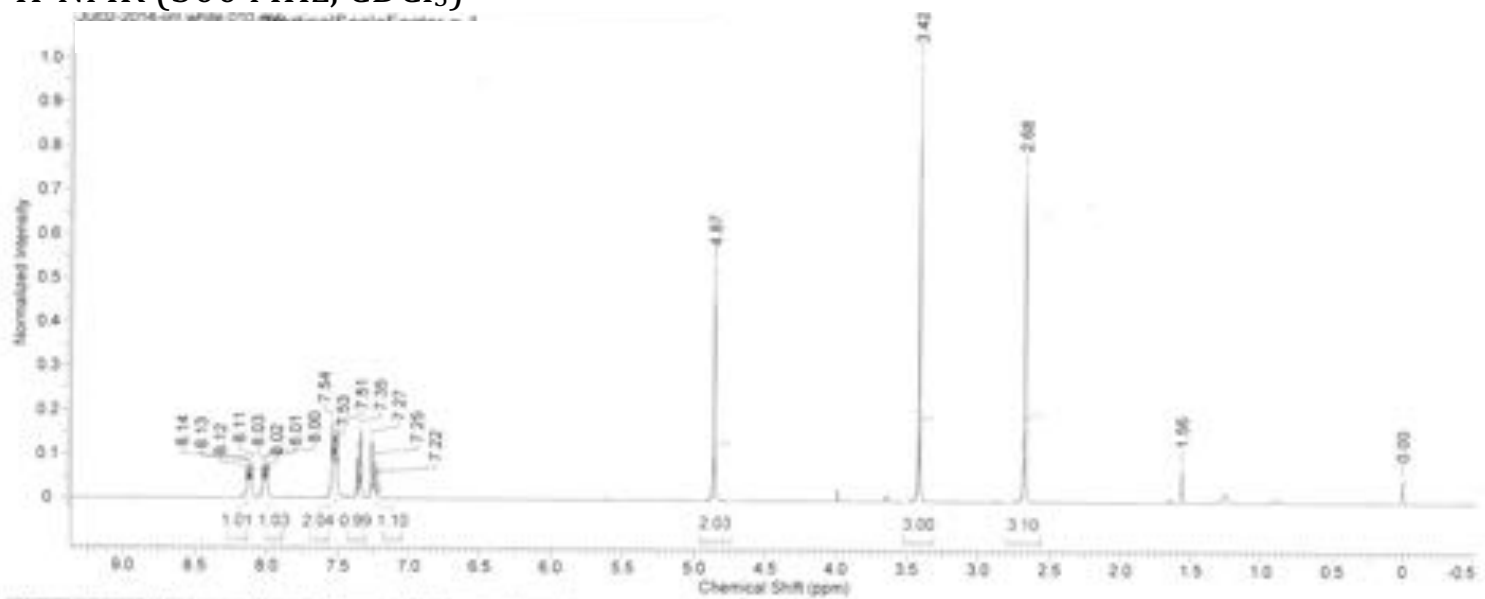

${ }^{13} \mathrm{C}$ UDEFT NMR (125 MHz, $\mathrm{CDCl}_{3}$ )

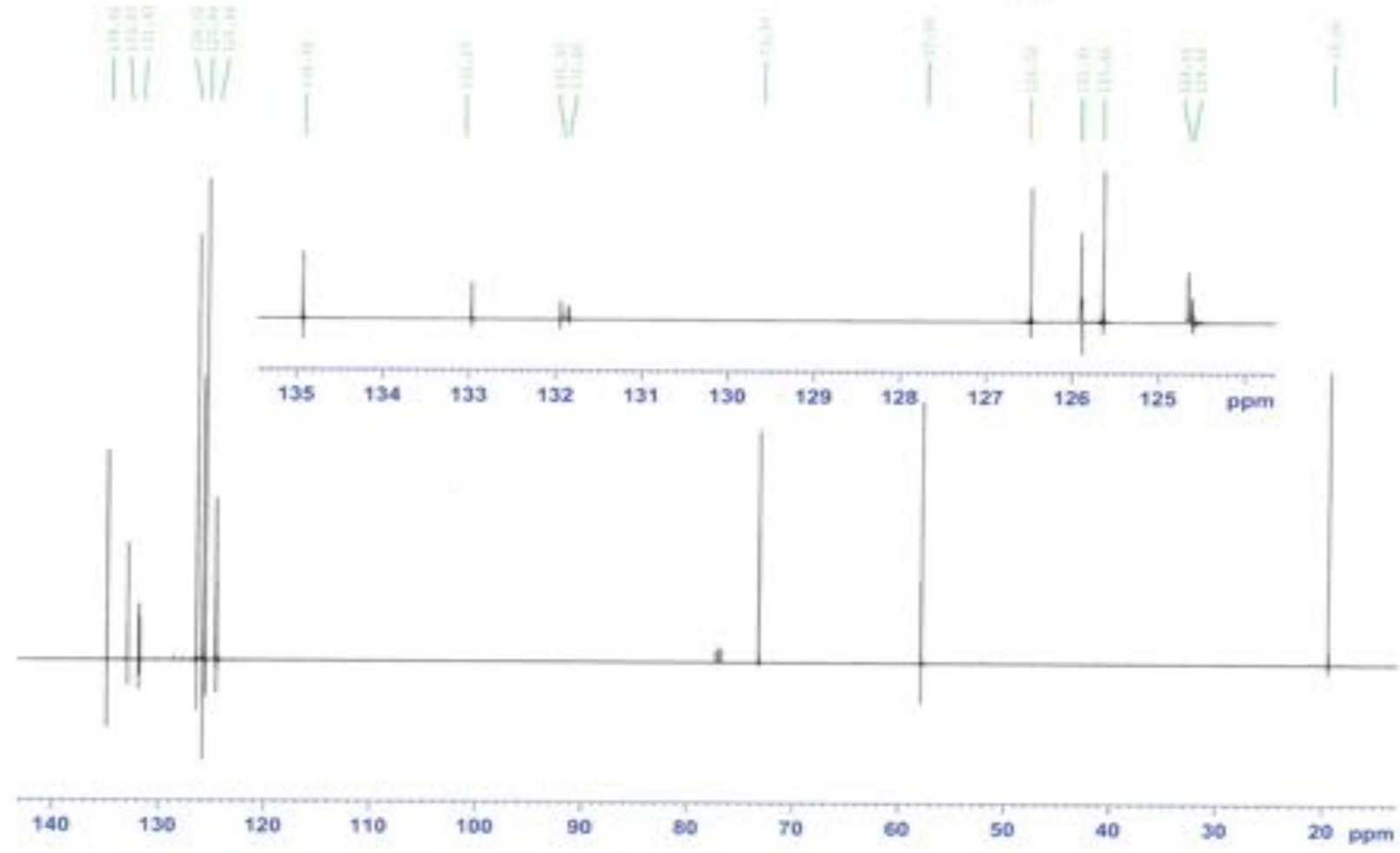


4-Methoxymethyl-1-naphthonitrile (6)

${ }^{1} \mathrm{H}-\mathrm{NMR}\left(500 \mathrm{MHz}^{\mathrm{CDCl}}{ }_{3}\right)$

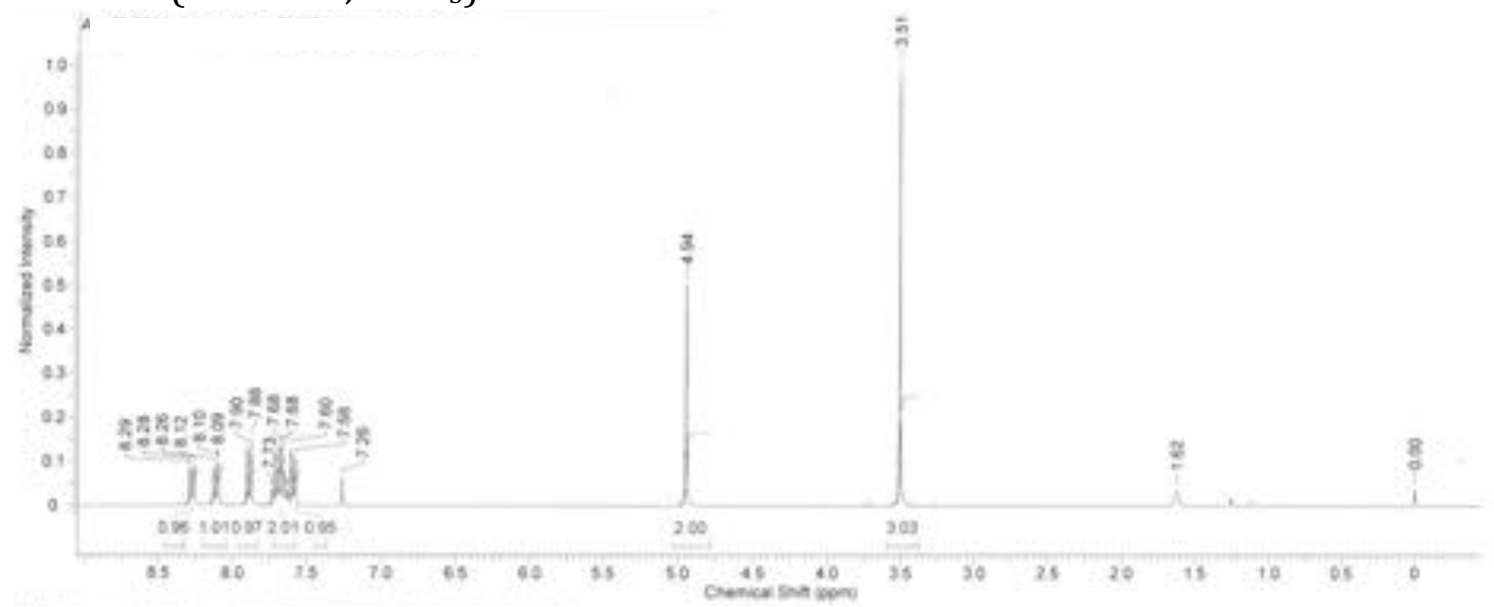

${ }^{13} \mathrm{C}$ UDEFT NMR $\left(125 \mathrm{MHz}, \mathrm{CDCl}_{3}\right)$
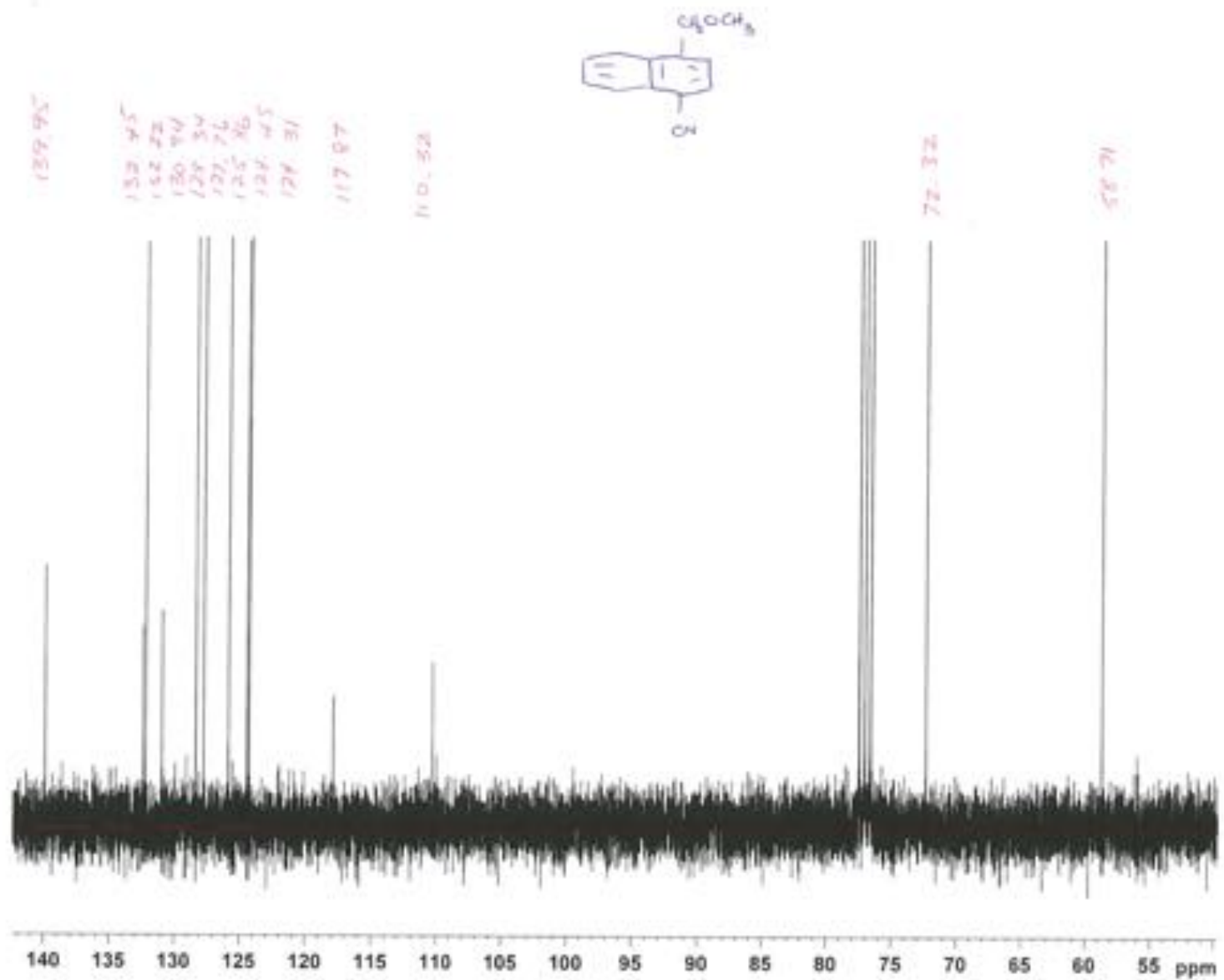